\title{
A critical discussion and practical recommendations on some issues relevant to the non-probabilistic treatment of uncertainty in engineering risk assessment
}

\author{
Nicola Pedroni ${ }^{1}$, Enrico Zio ${ }^{2}$, Alberto Pasanisi ${ }^{3}$, Mathieu Couplet ${ }^{4}$
}

${ }^{1}$ Corresponding author. Chair "System Science and the Energy challenge"-Fondation Electricité de France (EdF) at the Laboratoire Genie Industriel (LGI), CentraleSupélec, Université Paris-Saclay, Grande voie des Vignes, 92290 Chatenay-Malabry, France. E-mail:

nicola.pedroni@centralesupelec.fr

${ }^{2}$ Chair "System Science and the Energy challenge"-Fondation Electricité de France (EdF) at the Laboratoire Genie Industriel (LGI), CentraleSupélec, Université Paris-Saclay, Grande voie des Vignes, 92290 Chatenay-Malabry, France. E-mail: enrico.zio@ @entralesupelec.fr.

Also: Energy Department, Politecnico di Milano, Via Ponzio, 34/3 - 20133 Milano, Italy. E-mail: enrico.zio@polimi.it.

${ }^{3}$ EDF R\&D - EIFER, Emmy-Noether-Str. 11. 76131 Karlsruhe, Germany. E-mail address: alberto.pasanisi@eifer.org.

${ }^{4}$ Electricité de France, R\&D, 6 Quai Watier, 78400, Chatou, France. E-mail address: mathieu.couplet@edf.fr. 


\begin{abstract}
Models for the assessment of the risk of complex engineering systems are affected by uncertainties, due to the randomness of several phenomena involved and the incomplete knowledge about some of the characteristics of the system. The objective of the present paper is to provide operative guidelines to handle some conceptual and technical issues related to the treatment of uncertainty in risk assessment for engineering practice. In particular, the following issues are addressed: (1) quantitative modeling and representation of uncertainty coherently with the information available on the system of interest; (2) propagation of the uncertainty from the input(s) to the output(s) of the system model; (3) (Bayesian) updating as new information on the system becomes available; (4) modeling and representation of dependences among the input variables and parameters of the system model. Different approaches and methods are recommended for efficiently tackling each of the issues (1)-(4) above; the tools considered are derived from both classical probability theory as well as alternative, non-fully probabilistic uncertainty representation frameworks (e.g., possibility theory). The recommendations drawn are supported by the results obtained in illustrative applications of literature.
\end{abstract}

Keywords: uncertainty representation and propagation, Bayesian update, dependences. 


\section{INTRODUCTION}

The aim of this work is to critically address some conceptual and technical issues related to the treatment of uncertainty in risk assessment for engineering practice, with a particular focus on nonprobabilistic approaches. The motivation is the acknowledgement that the subject of risk nowadays plays a relevant role in the design, development, operation and management of components, systems and structures in many types of industry ${ }^{(1,2)}$. This is particularly true for civil, nuclear, aerospace and chemical systems that are safety-critical and must thus be designed and operated within a quantitative risk-informed approach aimed at systematically integrating deterministic and probabilistic analyses to obtain a rational decision on the utilization of resources for safety. In such rationalization, explicit consideration is given to the likelihood of accidents (scenarios) and to their potential consequences ${ }^{(3-12)}$.

In order to describe the physical phenomena that may lead to system failure, mathematical (risk) models are built that provide a representation of the real system dependent on a number of hypotheses and parameters. The risk model provides numerical outputs (e.g., relevant safety parameters) possibly to be compared with predefined numerical safety criteria for further guidance to risk-informed decision making processes $(4,7,10,13,14)$. However, in practice, not all the characteristics of the system under analysis can be fully captured in the risk model, which leads to uncertainty on both the values of the (input) parameters and on the hypotheses supporting the model structure $^{(3,5-7)}$ : such input uncertainty causes uncertainty in the model outputs and, thus, in the corresponding risk estimates. This output uncertainty must be estimated for a realistic quantification of the system behavior and associated risk, for use in decision making ${ }^{(3,15-17)}$.

In this paper, we focus on four relevant, conceptual and practical, issues on the treatment of uncertainty in the risk assessment of safety-critical engineering systems: 
1. The uncertainties in the model (input) parameters and hypotheses have to be first systematically identified and classified; then, they have to be quantitatively modeled and described by rigorous mathematical approaches coherently with the information available on the system. The key point is to guarantee that uncertainties are taken into account in a way that the knowledge relevant for the risk assessment process is represented in the most faithful manner ${ }^{(14,18-22)}$. For sake of simplicity, we will not deal here explicitly with uncertainties tainting the system's model itself. Whether this point is the object of many research and engineering works in the computer experiments community, e.g. Refs. 23-26, in engineering practice it is more common to separate the phases of assessing model's accuracy and propagating uncertainties from input to output variables ${ }^{(27)}$. See also the interesting and pragmatic viewpoint on this issue in Ref. 28.

2. The uncertainties in the input(s) have to be propagated onto the output(s) of the risk model (i.e., onto the risk measures), to provide the decision makers with a clearly risk-informed picture of the problem upon which they can confidently reason and deliberate ${ }^{(14,21,22,29)}$.

3. The quantitative representation of uncertainty needs to be updated, in a Bayesian framework, when new information/evidence (e.g., data) becomes available $\mathrm{e}^{(30-32)}$.

4. Possible dependences existing among the input parameters and variables of the system risk model need to be properly accounted for ${ }^{(33)}$. Actually, it is widely acknowledged that neglecting such dependences could lead to dramatic underestimations of the risk associated to the functioning of complex, safety-critical engineering systems $^{(34-37)}$ (e.g., underestimation of the magnitude of the consequences of an accident scenario).

For more than 30 years, a probabilistic framework has been embraced to address the process of risk assessment and the treatment of the associated uncertainties. The common term used is Probabilistic Risk Assessment (PRA, also referred to as Quantitative Risk Assessment-QRA) ${ }^{(4-7)}$. However, the 
purely probability-based approaches to risk and uncertainty analysis could be challenged under the common conditions of limited or poor knowledge, when the information available does not provide a strong basis for a specific probability assignment ${ }^{(29)}$. In this view, a broader risk description is sought where all the uncertainties are laid out 'plain and flat' with no additional information inserted in the analysis in the form of assumptions and hypotheses which cannot be proven right or wrong. This concern has sparked a number of investigations in the field of uncertainty representation and analysis, which has led to the developments of alternative (non-fully probabilistic) frameworks for describing uncertainties in risk assessments ${ }^{(18-21,28,38,39)}$, e.g., fuzzy set theory ${ }^{(40)}$, fuzzy probabilities ${ }^{(41)}$, random set theory ${ }^{(42)}$, evidence theory ${ }^{(33,43-46)}$, possibility theory ${ }^{(47-50)}$, probability bound analysis using probability-boxes (p-boxes) $)^{(37,51)}$, interval analysis ${ }^{(52,}$ ${ }^{53)}$ and interval probabilities ${ }^{(54)}$; notice that most of these theories can be included within the general common framework of imprecise probabilities ${ }^{(55-57)}$.

In this context, the main objective of the present paper is to show in a systematic and comprehensive framework how some conceptual and technical issues on the treatment of uncertainty in risk assessment (items 1.-4. above) can be effectively tackled outside the probabilistic setting: practically speaking, different approaches and methods will be recommended for efficiently addressing each of the issues 1.-4. listed above; classical probability theory tools as well as alternative, non-probabilistic ones (in particular, possibility theory) are considered. The recommendations are 'informed' by (i) a critical review of the literature approaches to solving the specific issues and (ii) the research work of the authors on addressing these issues: with respect to the latter item (ii), some of the considerations are based on results contained in articles previously published by the authors ${ }^{(58-61)}$; other conclusions are instead drawn from analyses originally presented in this paper (e.g., part of the work related to the issue of Bayesian updating). 
The remainder of the paper is organized as follows. In Section 2, risk assessment and uncertainty analysis are introduced. In Section 3, the four conceptual and technical issues related to uncertainty treatment in risk analysis mentioned above are presented and critically analyzed, and the relevant literature reviewed. In Section 4, techniques are recommended to effectively tackle such issues; results of the application of the proposed techniques to some case studies of literature are also shown. Finally, guidelines and recommendations are summarized in the concluding Section.

\section{UNCERTAINTY IN RISK ASSESSMENT}

The quantitative analyses of the phenomena occurring in many engineering systems and applications are based on mathematical models, which are translated into numerical computer codes for quantification ${ }^{(4,7,13)}$. In engineering practice, the mathematical models are not capable of capturing all the characteristics of the system under analysis ${ }^{(3,15)}$. This leads to uncertainty on both the values of the model input parameters/variables and on the hypotheses supporting the model structure. Such uncertainty propagates within the model and causes uncertainty in its outputs ${ }^{(16)}$. Formally, we consider a mathematical model $\boldsymbol{f}_{Z}(\boldsymbol{Y})$, which depends on the input quantities $\boldsymbol{Y}=\left\{Y_{1}\right.$, $\left.Y_{2}, \ldots, Y_{j}, \ldots, Y_{N}\right\}$ and on the (possibly implicit) function $f_{Z}(\cdot)$. The model is used to evaluate one or more output quantities $\boldsymbol{Z}=\left\{Z_{1}, Z_{2}, \ldots, Z_{l}, \ldots, Z_{O}\right\}$ of the system under analysis:

$\boldsymbol{Z}=\left\{Z_{1}, Z_{2}, \ldots, Z_{l}, \ldots, Z_{O}\right\}=\boldsymbol{f}_{Z}(\boldsymbol{Y})=\boldsymbol{f}_{Z}\left(Y_{1}, Y_{2}, \ldots, Y_{j}, \ldots, Y_{N}\right)$

By way of example, in the risk-based design of a flood protection dike the output quantity of interest may be represented by the water level of the river in proximity of a residential area ${ }^{(62)}$. In what follows, for the sake of simplicity of illustration and without loss of generality we consider only one (scalar) output $Z$, i.e., $\boldsymbol{Z}=\left\{Z_{1}, Z_{2}, \ldots, Z_{l}, \ldots, Z_{O}\right\} \equiv Z=f_{Z}(\boldsymbol{Y})$.

The uncertainty analysis of $Z$ requires an assessment of the uncertainties about $Y$ and their propagation through the model $f_{Z}(\cdot)$ to produce an assessment of the uncertainties about $Z$. 
In the context of risk assessment, uncertainty is conveniently distinguished into two different types: 'aleatory' (also known as 'objective', 'stochastic' or 'irreducible') and 'epistemic' (also known as 'subjective', 'state-of-knowledge' or 'reducible' $)^{(1-3,7,15,16,63-65)}$. Aleatory uncertainty is related to random variations, i.e., to the intrinsically random nature of several of the phenomena occurring during system operation. It concerns, for instance, the occurrence of the (stochastic) events that define various possible accident scenarios for a safety-critical system (e.g., a nuclear power plant) ${ }^{(6-}$ 10,66, 67), physical quantities like the maximal water flow of a river during a year, extreme events like earthquakes or natural processes like erosion and sedimentation ${ }^{(62,68)}$. Epistemic uncertainty is instead associated to the lack of knowledge about some properties and conditions of the phenomena underlying the behavior of the systems. This uncertainty manifests itself in the representation of the system behavior, in terms of both uncertainty in the model structure $f_{Z}(\cdot)$ and hypotheses assumed and parameter uncertainty in the (fixed but poorly known) values of the internal parameters $\boldsymbol{Y}$ of the model $^{(14,16,69)}$. While the first source of uncertainty has been widely investigated and more or less sophisticated methods have been developed to deal with it, research is still ongoing to obtain effective and agreed methods to handle the uncertainty related to the model structure ${ }^{(43,45)}$. See also Ref. 28 who distinguishes between model inaccuracies (the differences between $Z$ and $f_{Z}(\boldsymbol{Y})$ ), and model uncertainties due to alternative plausible hypotheses on the phenomena involved ${ }^{\mathrm{a}}$. In this paper, we are concerned only with the uncertainty in the model parameters $\boldsymbol{Y}=\left\{Y_{1}, Y_{2}, \ldots, Y_{j}, \ldots\right.$, $Y_{N}$ \}: an example is represented by the (imprecise) basic event probabilities in a fault tree ${ }^{(6,7,34,70)}$.

3 SOME ISSUES ON THE PRACTICAL TREATMENT OF UNCERTAINTIES IN ENGINEERING RISK ASSESSMENT:A CRITICAL LITERATURE SURVEY

\footnotetext{
${ }^{\text {a }}$ Notice that model uncertainty also includes the fact that the model could be too simplified and therefore would neglect some important phenomena affecting the final result. This latter type of uncertainty is sometimes identified independently from model uncertainty and is known as completeness uncertainty ${ }^{(6,7)}$.
} 
In Sections 3.1-3.4, four issues relevant to the treatment of uncertainty in engineering risk assessment are critically discussed, on the basis of the available literature on the subject.

\subsection{Quantitative modeling and representation of uncertainty coherently with the information available on the system}

Probability models are typically introduced to represent aleatory uncertainty: see, for example, the Poisson/exponential model for events randomly occurring in time (e.g., random variations of the operating state of a valve) $)^{(67,68)}$, the binomial model for describing the "failures on demand" of mechanical safety systems ${ }^{(7,71)}$ and the Gumbel model for the maximal water level of a river in a particular year ${ }^{(62)}$. Probability models constitute the basis for the statistical analysis of the data and information available on a system, and are considered essential for assessing the aleatory uncertainties and drawing useful insights on its random behavior ${ }^{(22)}$. They are also capable of updating the probability values, as new data and information on the system become available.

A probability model presumes some sort of model stability, by the construct of populations of similar units (in the Bayesian context, formally an infinite set of exchangeable random variables) ${ }^{(30,}$ ${ }^{72)}$. In this framework, the standard procedure for constructing probability models of random events and variables is as follows: (i) observe the process of interest over a finite period of time, (ii) collect data about the phenomenon, (iii) perform statistical analyses to identify the probability model (i.e., distribution) that best captures the variability in the available data and (iv) estimate the internal parameters of the selected probability model ${ }^{\mathrm{b}}(3,4,6,7,30,34,73,74)$. However, such 'presumed' model stability is often not fulfilled and the procedure (i)-(iv) above cannot be properly carried out ${ }^{(75)}$.

In the engineering risk assessment practical context, the situations are often unique, because the structures systems and components are, in the end, uniquely manufactured, operated and

\footnotetext{
${ }^{\mathrm{b}}$ In a frequentist view, the available data are interpreted as observable random realizations of an underlying, repeatable probabilistic model (e.g., a probability distribution) representing the aleatory phenomenon of interest, which can be approximated with increasing precision by the analyst as the size of the available data set increases ${ }^{(3)}$.
} 
maintained, so that their life realizations is not identical to any others. Then, the collection of repeated random realizations of the related random phenomena of interest (e.g., failure occurrences) means in reality the construction of fictional populations of non-existing similar situations. Then, probability models in general cannot be easily defined; in some cases, they cannot be meaningfully defined at all. For example, it makes no sense to define the (frequentist) probability of a terrorist $\operatorname{attack}^{(76)}$. In other cases, the conclusion may not be so obvious. For example, the (frequentist) probability of an explosion scenario in a process plant may be introduced in a risk assessment, although the underlying population of infinite similar situations is somewhat difficult to describe ${ }^{(21)}$. In addition, even when probability models with parameters can be established (justified) reflecting aleatory uncertainty, in many cases the amount of data available is insufficient for performing a meaningful statistical analysis on the random phenomenon of interest (e.g., because collecting this data is too difficult or costly); in other casas, the pieces of data themselves may be highly imprecise: in such situations, the internal parameters of the selected probability model cannot be estimated with sufficient accuracy and epistemic (state-of-knowledge) uncertainty is associated with them ${ }^{(49}$, 77). A full risk description needs to assess the (epistemic) uncertainties about these quantities. This framework of two hierarchical levels of uncertainty is referred to as "two-level" setting $(14,22,62,78)$. In the current risk assessment practice, the epistemic uncertainty in the parameters entering the (probability) models of random events is typically represented by (subjective) probability distributions within a Bayesian framework: subjective probability distributions capture the degree of belief of the analyst with respect to the values of the parameters entering the aleatory models, conditional on his/her background knowledge $\left.e^{(1-3,6,7,18,28,34,68,70,79-84}\right)$. However, the probabilitybased approach to epistemic uncertainty representation can be challenged by several practical and conceptual arguments. First of all, representing epistemic uncertainty by probability distributions (albeit subjective) amounts in practice to representing partial ignorance (imprecision) in the same way as randomness (variability) ${ }^{(49,77)}$ : then, the resulting distribution of the output can hardly be 
properly interpreted: "the part of the resulting variance due to epistemic uncertainty (that could be reduced) is unclear" ${ }^{\text {(77) }}$. Also, the fully probabilistic framework for assessing risk and uncertainties may be too narrow, as the subjective expert knowledge that the probability distributions are based on could be poor and/or even based on wrong assumptions, thus leading to conclusions that can mislead decision making. Actually, in the unique situations of risk assessment, the information available may not represent a sufficiently strong knowledge-basis for a specific probability assignment ${ }^{\mathrm{c}}$. Furthermore, in practical risk assessment and decision making contexts, "there are often many stakeholders and they may not be satisfied with a probability-based assessment based on subjective judgments made by one analysis group"(21): again, a broader risk description is sought. "It is true that adopting the subjective probability approach, probabilities can always be assigned, but the information basis supporting the assignments may not be reflected by the numbers produced. One may for example assess two situations both resulting in subjective probabilities equal to, e.g., 0.7, but in one case the assignment may be supported by substantial amount of relevant data, the other by no data at all"(21).

To overcome the above shortcomings of the fully probabilistic representation of uncertainty in risk assessment, alternative (non-fully probabilistic) approaches for representing and describing epistemic uncertainties in risk assessment have been suggested ${ }^{(18-21,37)}$, e.g., fuzzy set theory ${ }^{(40)}$, fuzzy probabilities ${ }^{(41)}$, random set theory ${ }^{(42)}$, Dempster-Shafer theory of evidence ${ }^{(33,43,44,46,85)}$, possibility theory ${ }^{(47-50)}$, interval analysis ${ }^{(52,53)}$, interval probabilities ${ }^{(54)}$ and probability bound analyses using p-boxes ${ }^{(37,51)}$.

In probability bound analysis, intervals are used for those parameters for which, due to ignorance, the analyst is not able or willing to assign a precise probability: rather, he/she prefers to describe such parameters only 'imprecisely' by means of a range of values, all of which coherent with the

\footnotetext{
${ }^{\mathrm{c}}$ Evidently, in those situations where the information is not of a type of "degree of belief" (in the sense of a subjective probability), one does not have the information needed to assign a specific probability: in those cases, the analyst may accept that and he/she is lead to interval probabilities or to develop such knowledge.
} 
information available and reflecting his/her (scarce) background knowledge on the problem; for the other components, traditional probabilistic analysis is carried out. This procedure results in a couple of extreme limiting Cumulative Distribution Functions (CDFs) (namely, a probability box or p-box) that bound above and below the "true" CDF of the quantity of interest. However, this way of proceeding results often in very wide intervals and the approach has been criticised for not providing the decision-maker with specific analyst and expert judgments about epistemic uncertainties $^{(18)}$. The other frameworks mentioned above allow for the incorporation and representation of incomplete information. Their motivation is to be able to treat situations where there is more information than that supporting just an interval assignment on an uncertain parameter, but less than that required to assign a single specific probability distribution.

All these theories produce epistemic-based uncertainty descriptions and in particular probability intervals. In fuzzy set theory membership functions are employed to express the degree of compatibility of a given numerical value to a fuzzy (i.e., vague, imprecisely defined) set (or interval). In possibility theory, uncertainty is represented by using a possibility distribution function that quantifies the degree of possibility of the values of a given uncertain parameter, say, $Y$. Formally, an application of possibility theory involves the specification of a pair $\left(U, \pi^{Y}\right)$ (called possibility space), where: (i) $U$ is a set that contains everything that could occur in the particular universe under consideration (e.g., it contains all the values that parameter $Y$ can assume); (ii) $\pi^{Y}$ is the possibility distribution function, defined on $U$ and such that $0 \leq \pi^{Y}(y) \leq 1$ for $y \in U$ and $\sup \left\{\pi^{Y}(y): y \in U\right\}=1^{(47-50)}$. Whereas in probability theory a single probability distribution function is introduced to define the probability of any interval (or event) $A$, in possibility theory one possibility function gives rise to a couple of probability bounds (i.e., upper and lower probabilities) for interval $A$, referred to as possibility and necessity measures and defined as $\Pi^{Y}(A)=\sup _{y \in A}\left\{\pi^{Y}(y)\right\}$ and $N^{Y}(A)=1-\sup _{y \notin A}\left\{\pi^{Y}(y)\right\}=1-\Pi^{Y}\left(A^{c}\right)$, respectively ${ }^{(47-50)}$. Finally, in evidence theory 
uncertainty is described by a so-called body of evidence, i.e., a list of focal sets/elements (e.g., intervals) each of which is assigned a probability (or belief) mass (so-called Basic Probability Assignment-BPA). Formally, an application of evidence theory involves the specification of a triple $(U, S, m)$ (called evidence space), where: (i) $U$ is a set that contains everything that could occur in the particular universe under consideration (namely, the sample space or universal set); (ii) $S$ is a countable collection of subsets of $U$ (i.e., the set of the so-called focal elements); (iii) $m$ is a function (i.e., the BPA) defined on subsets of $U$, such that: (i) $m(A)>0$, if $A \in S$; (ii) $m(A)=0$, if $A$ $\subset U$ and $A \notin S$, and (iii) $\sum_{A \in S} m(A)=1$. For a subset $A$ of $U, m(A)$ is a number characterizing the probability (or degree of belief) that can be assigned to A, but without any specification of how this degree of belief might be apportioned over $A$ : thus, it might be associated with any subset of $A$. In this respect, the function $m$ induces the so-called plausibility and belief measures that bound above and below the probability of a given set $A$ of interest: such measures are defined as $P l(A)=\sum_{B \cap A \neq \varnothing} m(B)$ and $\operatorname{Bel}(A)=\sum_{B \subset A} m(B)$, respectively. Measure $\operatorname{Bel}(A)$ can be viewed as the minimum degree of belief that must be associated with $A$ (i.e., it accounts for the evidence "supporting" A). Similarly, measure $P l(A)$ can be viewed as the maximum degree of belief that could be associated with $A$ (i.e., it accounts for the evidence "not contradicting" $A)^{(43,44,46,85,86)}$.

For the sake of completeness and precision, it is worth pointing out that the most of the theories mentioned above are 'covered' by the general common framework of imprecise probabilities ${ }^{(55-57)}$. Actually, as highlighted above, "a key feature of imprecise probabilities is the identification of bounds on probabilities for events of interest"(87). "The distance between the probability bounds reflects the indeterminacy in model specifications expressed as imprecision of the models. This imprecision is the concession for not introducing artificial model assumptions"(56). For further reflections on this subject, the reader is referred to Refs. $72,55,88$ and 89. 
It is worth admitting that these imprecise probability-based theories have not yet been broadly accepted for use in the risk assessment community. Till now, the development effort made on these subjects has mostly had a mathematical orientation, and it seems fair to say that no established framework presently exists for practical risk assessment based on these alternative theories ${ }^{(21)}$. Among the alternative approaches mentioned above, that based on possibility theory is by many considered one of the most attractive for extending the risk assessment framework in practice. In this paper, we focus on this approach for the following reasons: (i) the power it offers for the coherent representation of uncertainty under poor information (as testified by the large amount of literature in the field, see above); (ii) its relative mathematical simplicity; (iii) its connection with fuzzy sets and fuzzy logic, as conceptualized and put forward by Zadeh ${ }^{(90)}$ : actually, in his original view possibility distributions were meant to provide a graded semantics to natural language statements, which makes them particularly suitable for quantitatively translating (possibly vague, qualitative and imprecise) expert opinions; finally, (iv) the experience of the authors themselves in dealing and computing with possibility distributions ${ }^{(58-61)}$. One the other hand, it is worth remembering that possibility theory is only one of the possible "alternatives" to the incorporation of uncertainty into an analysis (see the approaches mentioned above).

\subsection{Propagation of uncertainty to the output of the system model}

The scope of the uncertainty analysis is the quantification and characterization of the uncertainty in the output $Z$ of the mathematical model $f_{Z}(\boldsymbol{Y})=f_{Z}\left(Y_{1}, Y_{2}, \ldots, Y_{j}, \ldots, Y_{N}\right)$ that derives from uncertainty in analysis inputs $\boldsymbol{Y}=\left\{Y_{1}, Y_{2}, \ldots, Y_{j}, \ldots, Y_{N}\right\}$ (see Section 2) ${ }^{(16)}$. In the light of the considerations reported in the previous Section 3.1, this requires the joint, hierarchical propagation of hybrid aleatory and epistemic uncertainties through the model $f_{Z}(\boldsymbol{Y})^{(67)}$.

When both aleatory and epistemic uncertainties in a two-level framework are represented by probability distributions, a two-level (or double loop) Monte Carlo (MC) simulation is usually 
undertaken to accomplish this $\operatorname{task}^{(62,74,91)}$ : the result is a 'bundle' of aleatory probability distributions, one for each realization of the epistemically-uncertain parameters.

Alternatively, when the epistemic uncertainties are represented by possibility distributions, the hybrid Monte Carlo (MC) and Fuzzy Interval Analysis (FIA) approach ${ }^{\mathrm{d}}$ is typically considered. In the hybrid MC-FIA method the MC technique ${ }^{(92)}$ is combined with the extension principle of fuzzy set theory ${ }^{(93-97)}$, within a "two-level" hierarchical setting $(49,58,60,98-100)$. This is done by: (i) FIA to process the uncertainty described by possibility distributions: in synthesis, intervals for the epistemically-uncertain parameters described by possibility distributions are identified by performing a repeated, level-wise interval analysis; (ii) MC sampling of the random variables to process aleatory uncertainty ${ }^{(49)}$ : given the intervals of the epistemically-uncertain parameters, families of probability distributions for the random variables are propagated through the model.

Instead, if the epistemic uncertainties are described within the framework of evidence theory, the Monte Carlo (MC)-based Dempster-Shafer (DS) approach employing Independent Random Sets (IRSs) $^{\mathrm{e}}$ is typically undertaken. In the MC-based DS-IRS method the focal sets (i.e., intervals) representing the epistemically-uncertain parameters are randomly and independently sampled by MC according to the corresponding probability (or belief) masses ${ }^{(39,101-103)}$.

In the present paper, particular focus is devoted to the MC-FIA approach: a detailed description of this technique and an illustrative application are reported in Section 4.2.

\subsection{Updating as new information becomes available}

In this Section, we address the issue of updating the representation of the epistemically-uncertain parameters of aleatory models (e.g., probability distributions), as new information/evidence (e.g., data) about the system becomes available.

\footnotetext{
${ }^{\mathrm{d}}$ In the following, this method will be referred to as "hybrid MC-FIA approach" for brevity.

e In the following, this method will be referred to as "MC-based DS-IRS approach" for brevity.
} 
The framework adopted is the typical Bayesian one - that is based on the well-known Bayes rule when epistemic uncertainties are represented by (subjective) probability distributions ${ }^{(30-32,73,104-107)}$. Alternatively, when the representation of epistemic uncertainty is non-probabilistic, other methods of literature can be undertaken ${ }^{(108)}$. In Ref. 109, a Generalized Bayes Theorem (GBT) has been proposed within the framework of evidence theory. In Refs. 110 and 111, a modification of Bayes theorem has been presented to account for the presence of fuzzy data and fuzzy prior Probability Distribution Functions (PDFs). In Refs. 112 and 113, a purely possibilistic counterpart of the classical, well-grounded probabilistic Bayes theorem has been proposed to update the possibilistic representation of the epistemically-uncertain parameters of (aleatory) probability distributions. Finally, Ref. 114 has introduced a hybrid probabilistic-possibilistic method that relies on the use of Fuzzy Probability Density Functions (FPDFs), i.e., PDFs with possibilistic (fuzzy) parameters (e.g., fuzzy means, fuzzy standard deviations, ...): it is based on the combination of: (i) Fuzzy Interval Analysis (FIA) to process the uncertainty described by possibility distributions and (ii) repeated Bayesian updating of the uncertainty represented by probability distributions.

In the present paper, the purely possibilistic Bayes' theorem is taken as reference: a detailed description of the approach and illustrative applications are reported in Section 4.3.

\subsection{Dependences among input variables and parameters}

Two types of dependence need to be considered in risk assessment ${ }^{(33)}$. The first type relates to the (dependent) occurrence of different (random) events (in the following, this kind of dependence will be referred to as 'objective' or 'aleatory'). An example of this objective (aleatory) dependence may be represented by the occurrence of multiple failures which result directly from a common or shared root cause (e.g., extreme environmental conditions, failure of a piece of hardware external to the system, or a human error): they are termed Common Cause Failures (CCFs) and typically can concern identical components in redundant trains of a safety system ${ }^{(7,115)}$; another example is that of 
cascading failures, i.e., multiple failures initiated by the failure of one component in the system, as a sort of chain reaction or domino effect ${ }^{(116,117)}$.

The second type refers to the dependence possibly existing between the estimates of the epistemically-uncertain parameters of the aleatory probability models used to describe random events/variables (in the following, this kind of dependence will be referred to as 'state-ofknowledge' or 'epistemic'). This state-of-knowledge (epistemic) dependence exists when the epistemically-uncertain parameters of aleatory models are estimated by resorting to dependent information sources (e.g., to the same experts/observers or to correlated data sets) ${ }^{(7,34)}$.

Considerable efforts have been done to address objective and state-of-knowledge dependences in risk analysis. In Ref. 118, objective dependencies among random events/variables have been treated by means of alpha factor models within the traditional framework of CCF analysis. In Refs. 33 and 119, the use of Frank copula and Pearson correlation coefficient has been proposed to describe a wide range of objective dependences among aleatory events/variables. In Ref. 120, (fuzzy) dependency factors are employed to model dependent events/variables. In Ref. 121 the rank correlation method has been proposed to characterize dependencies between epistemically uncertain variables. In Refs. 7 and 34, total (perfect) state-of-knowledge dependence among the failure rates of mechanical components has been modeled by imposing maximal correlation among the corresponding (subjective) probability distributions. In Refs. 122 and 123, state-of-knowledge dependences among the probabilities of the Basic Events (BEs) of a Fault Tree (FT) have been described by traditional correlation coefficients and propagated by the method of moments. In Ref. 124, statistical epistemic correlations have been modeled by resorting to the Nataf transformation within a traditional Monte Carlo Simulation (MCS) framework. In Refs. 59 and 91, the Dependency Bound Convolution (DBC) approach ${ }^{(33,125)}$ and the Distribution Envelop Determination (DEnv) method $^{(126-129)}$ have been adopted to account for all kinds of (possibly unknown) objective and epistemic dependences among correlated events/variables. 
In the present paper, particular focus is devoted to the DEnv method: a detailed description of the technique and an illustrative application to Fault Tree Analysis (FTA) are reported in Section 4.4.

\section{RECOMMENDATIONS FOR TACKLING THE CONCEPTUAL}

\section{AND TECHNICAL ISSUES ON UNCERTAINTY IN ENGINEERING RISK ASSESSMENT}

On the basis of the considerations made in Section 3, techniques are here recommended for tackling the four issues presented before. Guidelines on the recommended use of the techniques in practice are provided, with illustrative applications to simple risk assessment models.

\subsection{Quantitative modeling and representation of uncertainty coherently with the information available on the system}

In all generality, we consider an uncertain variable $Y$, whose (aleatory) uncertainty is described by a probability model, e.g., a $\operatorname{PDF} p^{Y}(y \mid \boldsymbol{\theta})$, where $\boldsymbol{\theta}=\left\{\theta_{1}, \theta_{2}, \ldots, \theta_{m}, \ldots, \theta_{P}\right\}$ is the vector of the corresponding internal parameters (see Section 3.1). In a two-level framework, the parameters $\boldsymbol{\theta}$ are themselves affected by epistemic uncertainty ${ }^{(62,78)}$. In the present work, we recommend to describe these epistemic uncertainties by the (generally joint) possibility distribution $\pi^{\theta}(\boldsymbol{\theta})$. A random variable $Y$ with possibilistic parameters $\boldsymbol{\theta}$ is referred to as a Fuzzy Random Variable (FRV) in the literature ${ }^{(49)}$. Details about FRVs are given in the following Section 4.1.1; then, the benefits of using a possibilistic description of epistemic uncertainty (instead of the classical, probabilistic one) are demonstrated by means of an illustrative example in Section 4.1.2.

\subsubsection{Recommended approach: Fuzzy Random Variables (FRVs)}

By way of example, we consider the uncertain variable $Y$ (e.g., the maximal water level of a river in a given year) described by a Gumbel probability model, i.e., $Y \sim p^{Y}(y \mid \boldsymbol{\theta})=p^{Y}(y \mid \gamma, \delta)=$ 
$1 / \delta \exp \left[-\left(\frac{y-\gamma}{\delta}+\exp \left(-\frac{y-\gamma}{\delta}\right)\right)\right]$. We suppose that parameter $\delta=\theta_{2}$ (i.e., the scale parameter) is known with absolute precision, i.e., it is a fixed point value $\left(\delta=\theta_{2}=100\right)$, whereas parameter $\gamma=\theta_{1}$ (i.e., the location parameter) is epistemically-uncertain. ${ }^{\mathrm{f}} \mathrm{We}$ consider, for the sake of the example, that the only information available on the value of the parameter $\gamma=\theta_{1}$ is that it ranges in the interval $\left[a_{\gamma}, b_{\gamma}\right]=[900,1300]$, with most likely value (i.e., mode) $c_{\gamma}=1100$. When the background knowledge on a parameter is partial like in the present case, the classical procedure for describing its uncertainty is to identify the corresponding maximum entropy PDF. However, this way of proceeding does not eliminate the fact that the information available on $\gamma=\theta_{1}$ is not sufficient for assigning a single specific PDF to describe the epistemic uncertainty in the parameter. In facts, such scarce information is compatible and consistent with a variety of PDFs (e.g., truncated normal, lognormal, triangular, ...) that obviously comprise also the maximum entropy one.

Alternatively, one of the ways of representing the uncertainty on $\gamma=\theta_{1}$ is offered by the framework of possibility theory ${ }^{(47-50)}$. For the simple numerical example considered above, a triangular possibility distribution $\pi^{\gamma}(\gamma)$ with core (i.e., vertex) $c_{\gamma}=1100$ and support (i.e., base) $\left[a_{\gamma}, b_{\gamma}\right]=$ [900, 1300] could be used (Figure 1, left) ${ }^{(50)}$ : indeed, it can be demonstrated that such possibility distribution encodes the family of all the probability distributions with mode $c_{\gamma}=1100$ and support $\left[a_{\gamma}, b_{\gamma}\right]=[900,1300]^{(43,47,50)}$ (obviously, this does not mean that the triangular possibility distribution is the only one able to describe such a probability family).

Actually, for a given set $S$ (i.e., of a given interval of values of parameter $\gamma$ ) the possibility function $\pi^{\gamma}(\gamma)$ gives rise to probability bounds (i.e., upper and lower probabilities), referred to as necessity and possibility measures $\left\{N^{\gamma}(S), \Pi^{\gamma}(S)\right\}$ and defined as $\Pi^{\gamma}(S)=\sup _{\gamma \in S}\left\{\pi^{\gamma}(\gamma)\right\}$ and

\footnotetext{
${ }^{\mathrm{f}}$ Obviously, in real risk assessment studies, a situation where one parameter of a given (aleatory) probability model is perfectly known and the other one is affected by significant epistemic uncertainty is far unlikely. However, notice that this example is here introduced only for the purpose of clearly and simply illustrating the basics of possibility theory.
} 
$N^{\gamma}(S)=1-\Pi^{\gamma}(\bar{S})=1-\sup _{\gamma \notin S}\left\{\pi^{\gamma}(\gamma)\right\}$, respectively, where $\bar{S}$ is the set (interval) complementary to $S$ on the axis of real numbers ${ }^{(43,47,50)}$. It can be demonstrated that the probability $P^{\gamma}(S)$ of the interval $S$ is bounded above and below by such necessity and possibility values, i.e., $N^{\gamma}(S) \leq P^{\gamma}(S) \leq \Pi^{\gamma}(S)$ : see Ref. 130 for a formal proof of this statement. Also, from the definitions of $\left\{N^{\gamma}(S), \Pi^{\gamma}(S)\right\}$ and referring to the particular set $S=(-\infty, \gamma]$, we can deduce the associated cumulative necessity and possibility measures $N^{\gamma}(S)=N^{\gamma}((-\infty, \gamma])$ and $\Pi^{\gamma}(S)=\Pi^{\gamma}((-\infty, \gamma])$, respectively (Figure 1, right). These measures can be interpreted as the limiting lower and upper bounds $\underline{F}^{\gamma}(\gamma)$ and $\bar{F}^{\gamma}(\gamma)$ to the "true" $\operatorname{CDF} F^{\gamma}(\gamma)=P((-\infty, \gamma])$, that we can build in coherence with the scarce information available on $\gamma$, i.e., only the mode and support. In other words, we can state that the triangular possibility distribution $\pi^{\gamma}(\gamma)$ of Figure 1 left produces a couple of CDFs (Figure 1 right), that bound the family of all the possible CDFs with mode $c_{\gamma}=1100$ and support $\left[a_{\gamma}, b_{\gamma}\right]=[900,1300]$ (see Refs. 43, 47 and 50 for a formal proof).

In order to provide an additional practical interpretation of the possibility distribution $\pi^{\gamma}(\gamma)$ of $\gamma=\theta_{1}$, we can define its so-called $\alpha$-cut sets (intervals) $A_{\alpha}^{\gamma}=\left\{\gamma: \pi^{\gamma}(\gamma) \geq \alpha\right\}$, with $0 \leq \alpha \leq 1$. For example, $A_{0.5}^{\gamma}=[1000,1200]$ is the set (interval) of $\gamma$ values for which the possibility function is greater than or equal to 0.5 (dashed segment in Figure 1, left). In the light of the discussion above, the $\alpha$-cut set $A_{\alpha}^{\gamma}$ of parameter $\gamma$ can be interpreted as the $(1-\alpha) \cdot 100 \%$ Confidence Interval (CI) for $\gamma$, i.e., the interval such that $P\left[\gamma \in A_{\alpha}^{\gamma}\right] \geq 1-\alpha$ : actually, $N\left(A_{\alpha}^{\gamma}\right) \leq P\left[\gamma \in A_{\alpha}^{\gamma}\right] \leq \Pi\left(A_{\alpha}^{\gamma}\right)$, which becomes, by definition of possibility and necessity measures, $1-\sup _{\gamma \notin A_{\alpha}^{\gamma}}\left\{\pi^{\gamma}(\gamma)\right\} \leq P\left[\gamma \in A_{\alpha}^{\gamma}\right] \leq$ $\sup _{\gamma \in A_{\alpha}^{\gamma}}\left\{\pi^{\gamma}(\gamma)\right\}$, i.e., $1-\alpha \leq P\left[\gamma \in A_{\alpha}^{\gamma}\right] \leq 1^{(47)}$ (Figure 1, left shows three CIs for $\alpha=0,0.5$ and 1 ).

In general, other techniques exist for constructing possibility distributions: for example, in Refs. 58 and 60 methods based on Chebyshev inequality are used to compute possibility distributions using 
estimated means and variances; finally, in Ref. 47 indications are provided to build possibility functions for uncertain parameters with known supports and means and/or quantiles.
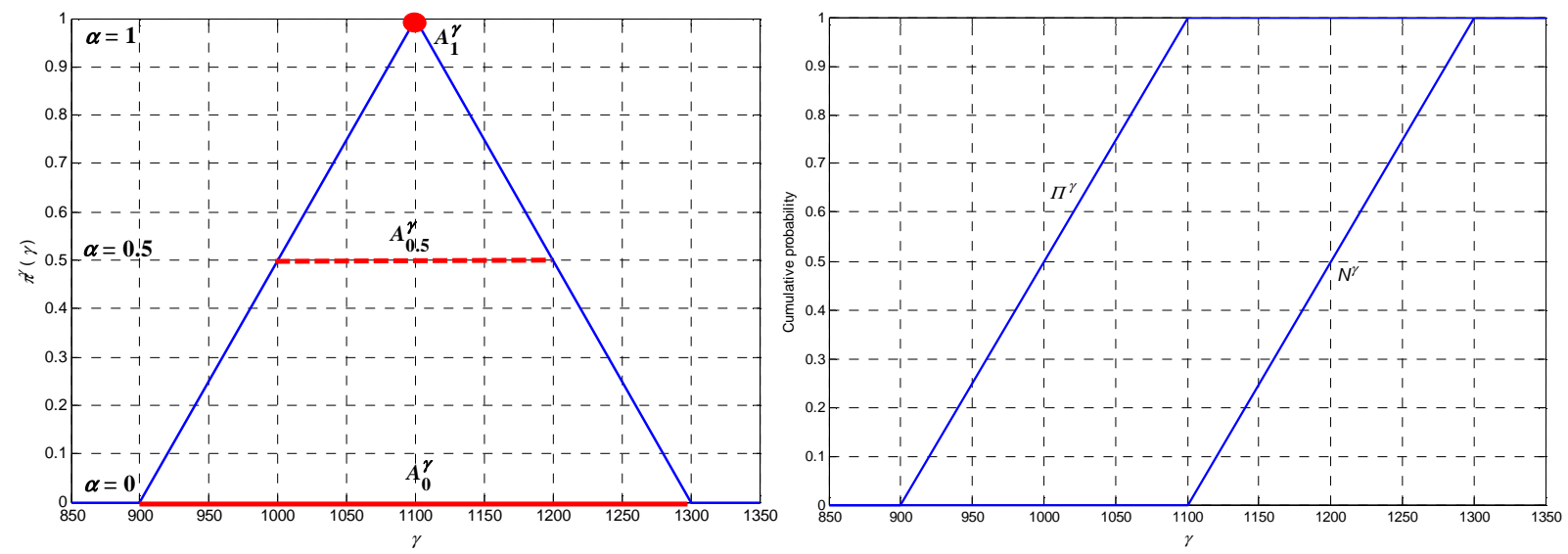

Figure 1. Left: triangular possibility distribution $\pi^{\gamma}(\gamma)$ of parameter $\gamma$. Right: bounding lower and upper CDFs for $\gamma, \underline{F}^{\gamma}(\gamma)=N^{\gamma}((-\infty, \gamma])$ and $\bar{F}^{\gamma}(\gamma)=\Pi^{\gamma}((-\infty, \gamma])$, respectively

As a result of the possibilistic description of the epistemically-uncertain parameter $\gamma$, for each possibility (resp., confidence) level $\alpha$ (resp., $1-\alpha$ ) in $[0,1]$, a family of PDFs (namely, $\left\{p^{Y}(y \mid \gamma, \delta)\right\}_{\alpha}$ ) can be generated also for random variable $Y$, by letting parameter $\gamma$ range within the corresponding $\alpha$-cut set $A_{\alpha}^{\gamma}$, i.e., $\left\{p^{Y}(y \mid \gamma, \delta)\right\}_{\alpha}=\left\{p^{Y}(y \mid \gamma, \delta): \gamma \in A_{\alpha}^{\gamma}, \delta=100\right\}$. By way of example, Figure 2, left, shows four PDFs belonging to the family $\left\{p^{Y}(y \mid \gamma, \delta)\right\}_{\alpha}$ for $\alpha=0$ (solid lines) and $\alpha=0.5$ (dashed lines). In the same way, a bundle of CDFs for $Y$, namely $\left\{F^{Y}(y \mid \gamma, \delta)\right\}_{\alpha}=$ $\left\{F^{Y}(y \mid \gamma, \delta): \gamma \in A_{\alpha}^{\gamma}, \sigma=100\right\}$, can be constructed, which is bounded above and below by $\bar{F}_{\alpha}^{Y}(y)=\sup _{\gamma \in A_{\alpha}^{\gamma}}\left\{F^{Y}(y \mid \gamma, \delta=100)\right\}$ and $\underline{F}_{\alpha}^{Y}(y)=\inf _{\gamma \in A_{\alpha}^{\gamma}}\left\{F^{Y}(y \mid \gamma, \delta=100)\right\}$, respectively. Since $\pi^{\gamma}(\gamma)$ can be interpreted as a set of nested CIs for parameter $\gamma$ (see above), it can be argued that the $\alpha$-cuts of $\pi^{\gamma}(\gamma)$ induce also a set of nested pairs of CDFs $\left\{\left(\underline{F}_{\alpha}^{Y}(y), \bar{F}_{\alpha}^{Y}(y)\right): 0 \leq \alpha \leq 1\right\}$ which bound the "true" CDF $F^{Y}(y)$ with confidence larger than or equal to $(1-\alpha)$, i.e., $P\left[\underline{F}_{\alpha}^{Y}(y) \leq F^{Y}(y) \leq \bar{F}_{\alpha}^{Y}(y)\right] \geq 1-\alpha$, with $0 \leq \alpha \leq 1^{(49,94,95)}$. Using the jargon of evidence theory ${ }^{(44-46)}, \bar{F}_{\alpha}^{Y}(y)$ and $\underline{F}_{\alpha}^{Y}(y)$ are often referred to as the plausibility and belief functions (of level $\alpha$ ) of the set $S=(-\infty, y]$, i.e., 
$\bar{F}_{\alpha}^{Y}(y)=P l_{\alpha}^{Y}(S)$ and $\underline{F}_{\alpha}^{Y}(y)=\operatorname{Bel}_{\alpha}^{Y}(S)$, respectively (see Section 3.1). For illustration purposes, Figure 2, right, shows $P l_{\alpha}^{Y}(S)$ and $\operatorname{Bel}_{\alpha}^{Y}(S), S=(-\infty, y]$, built in correspondence of the $\alpha$-cuts of level $\alpha=0$ (solid lines), 0.5 (dashed lines) and 1 (dot-dashed line) of $\pi^{\gamma}(\gamma)$ (Figure 1, left).

Finally, the set of nested pairs of $\mathrm{CDFs}\left\{\left(\operatorname{Bel}_{\alpha}^{Y}(S), P l_{\alpha}^{Y}(S)\right): 0 \leq \alpha \leq 1\right\}, S=(-\infty, y]$, can be synthesized into a single pair of plausibility and belief functions as $P l^{Y}(S)=\int_{0}^{1} P l_{\alpha}^{Y}(S) d \alpha$ and $\operatorname{Bel}^{Y}(S)=\int_{0}^{1} \operatorname{Bel}_{\alpha}^{Y}(S) d \alpha$, respectively (dotted lines in Figure 2, right): in other words, $P l^{Y}(S)$ and $\operatorname{Bel}^{Y}(S)$ are obtained by averaging the nested plausibility and belief functions generated at different possibility levels $\alpha \in[0,1]$. The plausibility and belief functions $\mathrm{Pl}^{Y}(S)$ and $\mathrm{Bel}^{Y}(S)$ are shown to represent the "best bounds" for the "true" $\operatorname{CDF} F^{Y}(y)$ of $Y^{(49,94,95,131)}$.
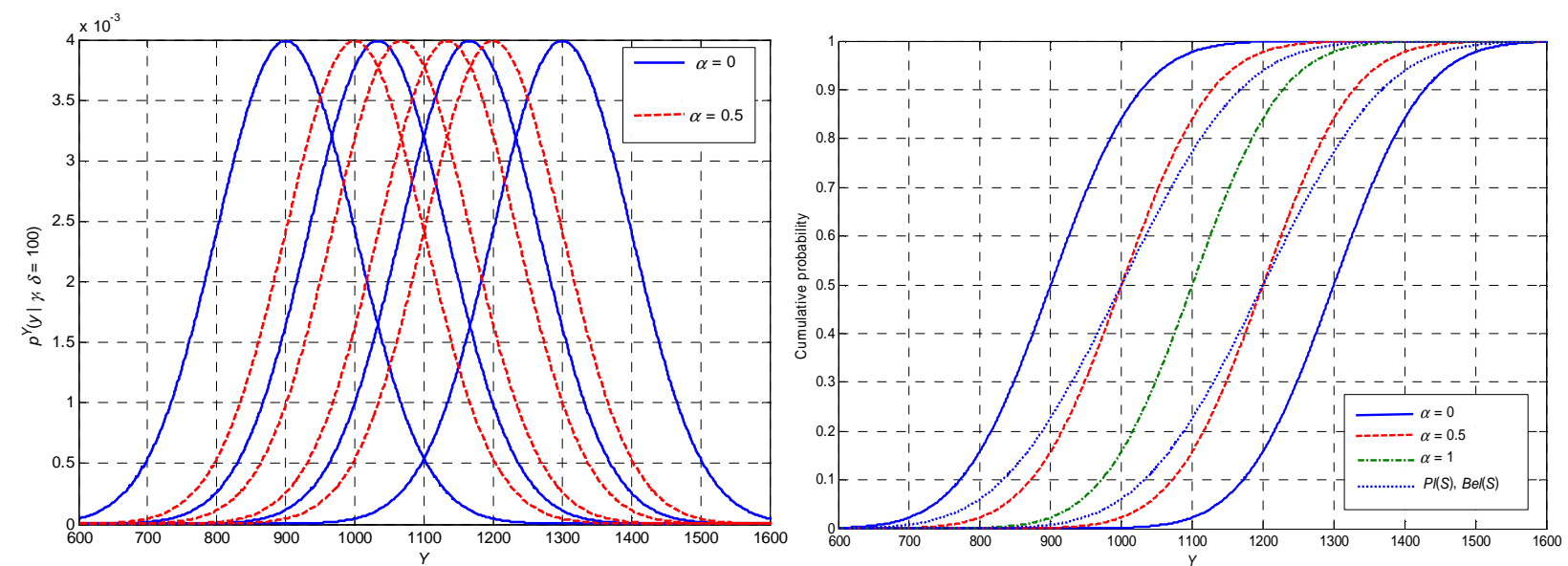

Figure 2. Example of Fuzzy Random Variable (FRV)

\subsubsection{Illustrative example}

The benefits coming from the use of the hybrid probabilistic and possibilistic FRV approach illustrated in the previous Section 4.1.1 are here shown by comparison with a traditional, purely probabilistic two-level framework, where the epistemically-uncertain parameter $\gamma$ of the Gumbel probability model is itself described by a single $\operatorname{PDF} p^{\gamma}(\gamma)$; notice that the results reported hereafter are presented for the first time in this paper. In order to perform a fair comparison between 
the two approaches, a sample from the PDF $p^{\gamma}(\gamma)$ here employed is obtained by applying the principle of insufficient reason ${ }^{(132)}$ to the possibility distribution $\pi^{\gamma}(\gamma)$ of Figure 1 , left. The procedure for obtaining such a sample is ${ }^{(132,133)}$ : (i) draw a random realization $\alpha^{*}$ for $\alpha$ in $[0,1)$ and consider the $\alpha$-cut level $A_{\alpha^{*}}^{\gamma}=\left[\underline{\gamma}_{\alpha^{*}}, \bar{\gamma}_{\alpha^{*}}\right]=\left\{\gamma: \pi^{\gamma}(\gamma) \geq \alpha^{*}\right\}$; (ii) sample a random realization $\gamma^{*}$ for $\gamma$ from a uniform probability distribution on $A_{\alpha^{*}}^{\gamma}$. Other techniques for the transformation of possibility distributions into PDFs can be found in Refs. 60, 132, 134 and 135.

In order to highlight the effects of different representations of epistemic uncertainty, we analyze the 95-th quantile $Y^{0.95}$ of the uncertain variable $Y$. Figure 3 shows the corresponding bounding CDFs $\bar{F}^{Y^{0.95}}\left(y^{0.95}\right)$ and $\underline{F}^{Y^{0.95}}\left(y^{0.95}\right)\left(y^{0.95} \in \Re\right)$ produced using a possibilistic (solid lines) representation of the epistemically-uncertain parameter $\gamma$ together with the single $\operatorname{CDF} F^{Y^{0.95}}\left(y^{0.95}\right)\left(y^{0.95} \in \mathfrak{R}\right)$ obtained by resorting to a probabilistic description of parameter $\gamma$ (dashed line). In this respect, it is important to remember that in a two-level hierarchical framework of uncertainty representation, the quantiles of an uncertain variable $Y$ are not fixed point values, but rather they are epistemicallyuncertain variables. In particular, if epistemic uncertainty is represented by probability distributions, then the quantiles of $Y$ are correspondingly described by probability distributions. In the same way, when epistemic uncertainty is represented by possibility distributions, then the quantiles are described by possibility distributions ${ }^{(58)}$. In this latter case, a couple of bounding CDFs (i.e., of cumulative possibility and necessity measures) can be associated to the corresponding quantiles using the formulas introduced in Section 4.1.1. The advantage of using a non-probabilistic representation of epistemic uncertainty lies in the possibility of providing bounds on the estimates of the 95-th quantile (in the light of the scarce information available on the variable). For example, let us refer to the quantitative indicator $P\left[Y^{0.95}>y^{0.95^{*}}\right]$, i.e., the probability that $Y^{0.95}$ exceeds a given safety threshold $y^{0.95^{*}}(=1400$ in this case): the point estimate provided by the purely 
probabilistic approach is 0.0285 , whereas the interval produced by the hybrid FRV approach is [0, 0.3225]. If a faithful description of the (scarce and imprecise) information available on the variable $Y$ leads to $P\left[Y^{0.95}>y^{0.95 *}\right]=[0,0.3225]$, then the analyst cannot exclude that in reality such exceedance probability is equal to the highest "possible" value obtained (i.e., 0.3225) ${ }^{\mathrm{g}}$. In this light, it can be seen that the upper bound 0.3225 of the interval $[0,0.3225]$ (representing a conservative assignment of the exceedance probability "informed" by a faithful representation of the imprecision related to $\gamma$ ) is about 11 times larger than the corresponding point value generated by the purely probabilistic method ${ }^{\mathrm{h}}$. This means that if we base our analysis on an inappropriately precise assumption (i.e., on the selection of a specific probability distribution even in presence of scarce information), we may significantly underestimate risk (for example, the probability of a given accident and/or the severity of the corresponding consequences). Instead, using families of probability distributions (within the framework, e.g., of possibility theory) implicitly introduce a sort of "informed conservatism" into the analysis, which should lead the decision maker to be "safely"-coherent with his/her limited and/or imprecise background knowledge. ${ }^{\mathrm{i}}$

On the other hand, it has to be acknowledged that: (i) a poorly done possibilistic analysis can be just as misleading as a poorly done probabilistic analysis; (ii) even if possibility theory constitutes a "rigorous" tool for transforming the available information into an uncertainty representation, such uncertainty description remains, to a certain degree, subjective: for example, "it requires additional judgments in the specification of the possibility function"(21); (iii) as argued in Ref. 18, even if a possibilistic analysis "meets to a large extent the ambition of objectivity by trying to reduce the

\footnotetext{
g A remark is in order with respect to this sentence and to the existence "in reality" of a "true value" of the probability. For subjective probabilities such "true" values have no meaning. Instead "true" values can be obviously defined for the frequentist probabilities. In the present paper we adopt a two-level, hierarchical framework to model uncertainty (also called probability-of-frequency approach): thus, a true value of the exceedance probability can be defined.

${ }^{\mathrm{h}}$ In this paper, a given (probability or consequence) estimate is considered in a broad sense more "conservative" than another one, if it implies and leads to a higher level of risk. However, since a discussion on the concept of "conservatism" goes beyond the scopes of the present paper, further details are not given here for brevity: the interested reader is referred to, e.g., Ref. 136 and references therein.

${ }^{i}$ In other words, in line with Ref. 18, we may say that such non-probabilistic approaches can be considered "objective" tools for transforming the available information into an uncertainty representation, in the sense that they try to avoid the use of "subjective judgements and assumptions" in the analysis.
} 
amount of arbitrary assumptions made in the analyses, it does not provide the decision maker with specific scientific judgments about epistemic uncertainties from qualified analysts and experts". Actually, "expressing epistemic uncertainties means a degree of subjectivity, and decision making normally needs to be supported by qualified judgments" ${ }^{(18)}$. In this respect, sometimes the bounds provided by a possibilistic analysis may be considered rather "non-informative and the decision maker would ask for a more refined assessment: in such cases, it is expected that the analysts are able to give some qualified judgments, so that a more precise assessment can be obtained"(18).

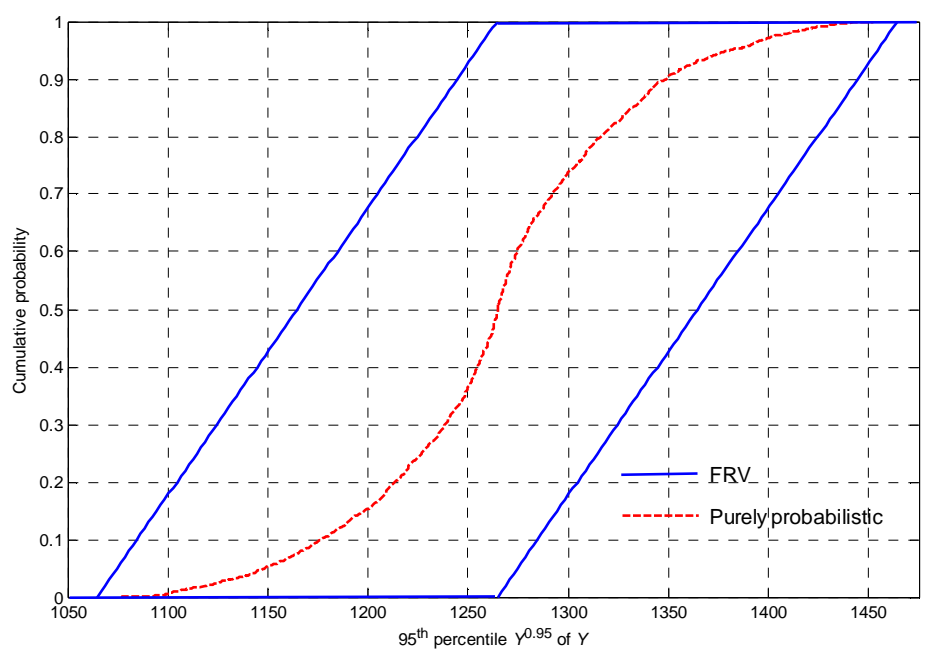

Figure 3. Epistemic distributions of the 95-th quantile $Y^{0.95}$ of $Y \sim G u m(\gamma, \delta)$ obtained through a possibilistic (solid lines) and probabilistic (dashed line) representation of parameter $\gamma$

\subsection{Propagation of uncertainty to the output of the system model}

With reference to the model function $Z=f_{Z}(\boldsymbol{Y})=f_{Z}\left(Y_{1}, Y_{2}, \ldots, Y_{j}, \ldots, Y_{N}\right)(1)$, we consider $N$ input variables $\boldsymbol{Y}=\left\{Y_{1}, Y_{2}, \ldots, Y_{j}, \ldots, Y_{N}\right\}$ affected by hybrid aleatory and epistemic uncertainty and represented by PDFs $\left\{p_{Y_{j}}\left(y_{j} \mid \boldsymbol{\theta}_{j}\right): j=1,2, \ldots, N\right\}$ with epistemically-uncertain parameters $\left\{\boldsymbol{\theta}_{j}: j=\right.$ $1,2, \ldots, N\}$ described by possibility distributions $\boldsymbol{\pi}^{\boldsymbol{\theta}_{j}}\left(\boldsymbol{\theta}_{j}\right)$. In order to jointly propagate these mixed uncertainty representations, we recommend the hybrid Monte Carlo (MC) and Fuzzy Interval Analysis (FIA) approach detailed in Section 4.2.1; the effectiveness of the proposed approach is assessed by comparison with other uncertainty propagation techniques in Section 4.2.2.

\subsubsection{Recommended approach: Monte Carlo (MC) and Fuzzy Interval Analysis (FIA)}


The hybrid MC and FIA approach combines the MC technique ${ }^{(92)}$ with the extension principle of fuzzy set theory ${ }^{(93-97,135)}$ by means of the following main steps ${ }^{(49,58,60,98-100)}$ :

1. $\operatorname{set} \alpha=0$ (outer loop, processing epistemic uncertainty by FIA);

2. select the $\alpha$-cut sets $A_{\alpha}^{\boldsymbol{\theta}_{j}}$ of the $N$ (joint) possibility distributions $\boldsymbol{\pi}^{\boldsymbol{\theta}_{j}}\left(\boldsymbol{\theta}_{j}\right)$ of the epistemically-uncertain parameters vectors $\left\{\boldsymbol{\theta}_{j}: j=1,2, \ldots, N\right\}$;

3. letting parameters $\boldsymbol{\theta}_{j}$ range within the corresponding $\alpha$-cuts $A_{\alpha}^{\boldsymbol{\theta}_{j}}$, 'generate' families of CDFs $\left\{F^{Y_{j}}\left(y \mid \boldsymbol{\theta}_{j}\right)\right\}_{\alpha}=\left\{F^{Y_{j}}\left(y \mid \boldsymbol{\theta}_{j}\right): \boldsymbol{\theta}_{j} \in A_{\alpha}^{\boldsymbol{\theta}_{j}}\right\}$ for the "hybrid" input variables $Y_{j}, j=1, \ldots, N$

4. propagate the (input) families of CDFs $\left\{F^{Y_{j}}\left(y \mid \boldsymbol{\theta}_{j}\right)\right\}_{\alpha}$ through the mathematical model $Z=f\left(Y_{1}, Y_{2}, \ldots, Y_{n}\right)$ by standard MC sampling to obtain the (output) family of CDFs $\left\{F^{Z}(z)\right\}_{\alpha}$ for $Z$ (inner loop, processing aleatory uncertainty by standard MC simulation);

5. identify the limiting CDFs (that bound above and below the "true" $\operatorname{CDF} F^{Z}(z)$ with confidence $(1-\alpha) \cdot 100 \%)$ as those that 'envelop' the family $\left\{F^{Z}(z)\right\}_{\alpha}$, i.e., $\bar{F}_{\alpha}^{Z}(z)=$ $P l_{\alpha}^{Z}((-\infty, z])=\max \left\{\bar{F}_{\alpha}^{Z}(z)\right\}$ and $\underline{F}_{\alpha}^{Z}(z)=\operatorname{Bel}_{\alpha}^{Z}((-\infty, z])=\min \left\{\bar{F}_{\alpha}^{Z}(z)\right\}$, respectively;

6. if $\alpha<1$, then set $\alpha=\alpha+\Delta \alpha$ (e.g., $\Delta \alpha=0.05$ in this paper) and return to step 2. above; otherwise, stop the algorithm.

The output of the algorithm is thus represented by nested sets of plausibility and belief functions $\left\{\left(\operatorname{Bel}_{\alpha}^{Z}(A), P l_{\alpha}^{Z}(A)\right): 0 \leq \alpha \leq 1\right\}, A=(-\infty, z]$ : these sets of functions can then be synthesized into a single pair of plausibility and belief functions, $\operatorname{Pl}^{Z}(A)$ and $\operatorname{Bel}^{Z}(A), A=(-\infty, z]$, as described in Section 4.1.1.

It is worth noting that performing an interval analysis on $\alpha$-cuts assumes total dependence among the epistemically-uncertain parameters. Actually, this procedure implies strong dependence among the information sources (e.g., the experts or observers) that supply the input possibility 
distributions, because the same confidence level $(1-\alpha)$ is chosen to build the $\alpha$-cuts for all the epistemically-uncertain parameters $^{(48)}$ (see Section 4.4 for further discussions on dependence).

\subsubsection{Illustrative examples}

In what follows, we report some of the results obtained in a previous work by some of the authors $^{(58)}$, in which the hybrid MC-FIA approach (Section 4.2.1) is used to propagate mixed probabilistic and possibilistic uncertainties through mathematical models. The effectiveness of the proposed technique is shown by means of a comparison with the MC-based Dempster-Shafer (DS) approach employing Independent Random Sets (IRSs), where the possibility distributions describing the epistemically-uncertain parameters are discretized into focal sets that are randomly and independently sampled by MC (see Section 3.2). Such discretization requires the following steps $^{(48)}$ : (i) determine $N_{e}$ (nested) focal sets for the generic possibilistic parameter $\theta$ as the $\alpha$-cuts $A_{\alpha_{t}}=\left[\underline{\theta}_{\alpha_{t}}, \bar{\theta}_{\alpha_{t}}\right], t=1,2, \ldots, N_{e}$, with $\alpha_{1}=1>\alpha_{2}>\ldots>\alpha_{N_{e}-1}>\alpha_{N_{e}}=0$; (ii) build the probability mass distribution of the focal sets by assigning $m_{\alpha_{t}}=\Delta \alpha_{t}=\alpha_{t}-\alpha_{t+1}$. In this paper, $N_{e}=21$ and $m_{\alpha t}=0.05$ are chosen for the sake of comparison with the hybrid MC-FIA approach (see Section $4.2 .1) .(58,60)$

For the exemplification, we consider the mathematical model $Z_{1}=f_{Z l}\left(Y_{1}, Y_{2}, Y_{3}\right)$ already presented in Ref. 58: the uncertain inputs $Y_{1}, Y_{2}$ and $Y_{3}$ are described by lognormal probability distributions with (triangular) possibilistic parameters. Figure 4 shows the plausibility and belief functions, $\operatorname{Pl}^{Z_{1}}\left(\left(-\infty, z_{1}\right]\right)=\bar{F}^{Z_{1}}\left(z_{1}\right)$ and $\operatorname{Bel}^{Z_{1}}\left(\left(-\infty, z_{1}\right]\right)=\underline{F}^{Z_{1}}\left(z_{1}\right)$ of the model outputs $Z_{1}$ produced by the hybrid MC-FIA (solid lines) and MC-based DS-IRS (dashed lines) approaches ${ }^{(58)}$. The results are very similar, i.e., in the present case, the effect of the different uncertainty propagation method is not evident ${ }^{(58,60)}$. Then, in order to highlight the effects of the different uncertainty propagation approaches, the upper and lower CDFs, $\bar{F}^{Z_{1}^{0.95}}\left(z_{1}^{0.95}\right)$ and $\underline{F}^{Z_{1}^{0.95}}\left(z_{1}^{0.95}\right)$, respectively, of the 95-th 
quantile $Z_{1}^{0.95}$ of $Z_{1}$ are further analyzed. Figure 5, left, shows the bounding CDFs for $Z_{1}^{0.95}$ produced by the hybrid MC-FIA (solid lines) and the MC-based DS-IRS (dashed lines) approach; for illustration purposes, Figure 5, right, shows the possibility distributions $\pi^{Z_{1}^{0.95}}\left(z_{1}^{0.95}\right)$ that corresponds to the CDFs by means of the relations $\bar{F}^{Z_{1}^{0.95}}\left(z_{1}^{0.95}\right)=\sup _{Z_{1}^{0.95} \leq z_{1}^{0.95}}\left\{\pi_{1}^{Z_{1}^{0.95}}\left(z_{1}^{0.95}\right)\right\}$ and $\underline{F}^{Z_{1}^{0.95}}\left(z_{1}^{0.95}\right)=1-\sup _{Z_{1}^{0.95}>z_{1}^{0.95}}\left\{\pi_{1}^{Z_{1}^{0.95}}\left(z_{1}^{0.95}\right)\right\}{ }^{(48)}$

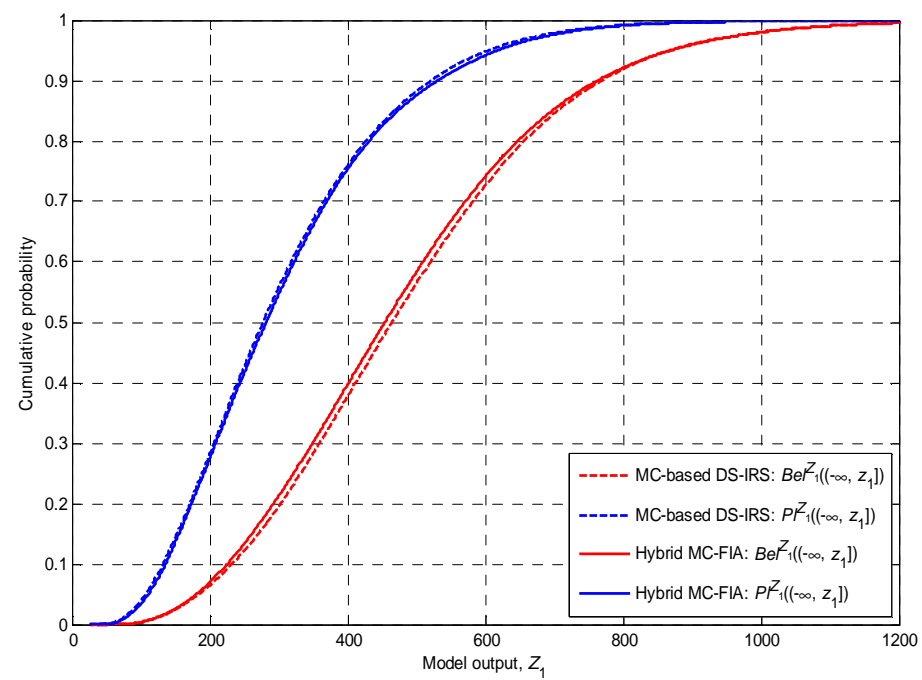

Figure 4. Plausibility and belief functions $\operatorname{Pl}^{Z_{1}}\left(\left(-\infty, z_{1}\right]\right)$ and $\mathrm{Bel}^{Z_{1}}\left(\left(-\infty, z_{1}\right]\right)$ of model output $Z_{1}$ obtained by the hybrid MC-FIA (solid lines) and MC-based DS-IRS (dashed lines) approaches ${ }^{(58)}$
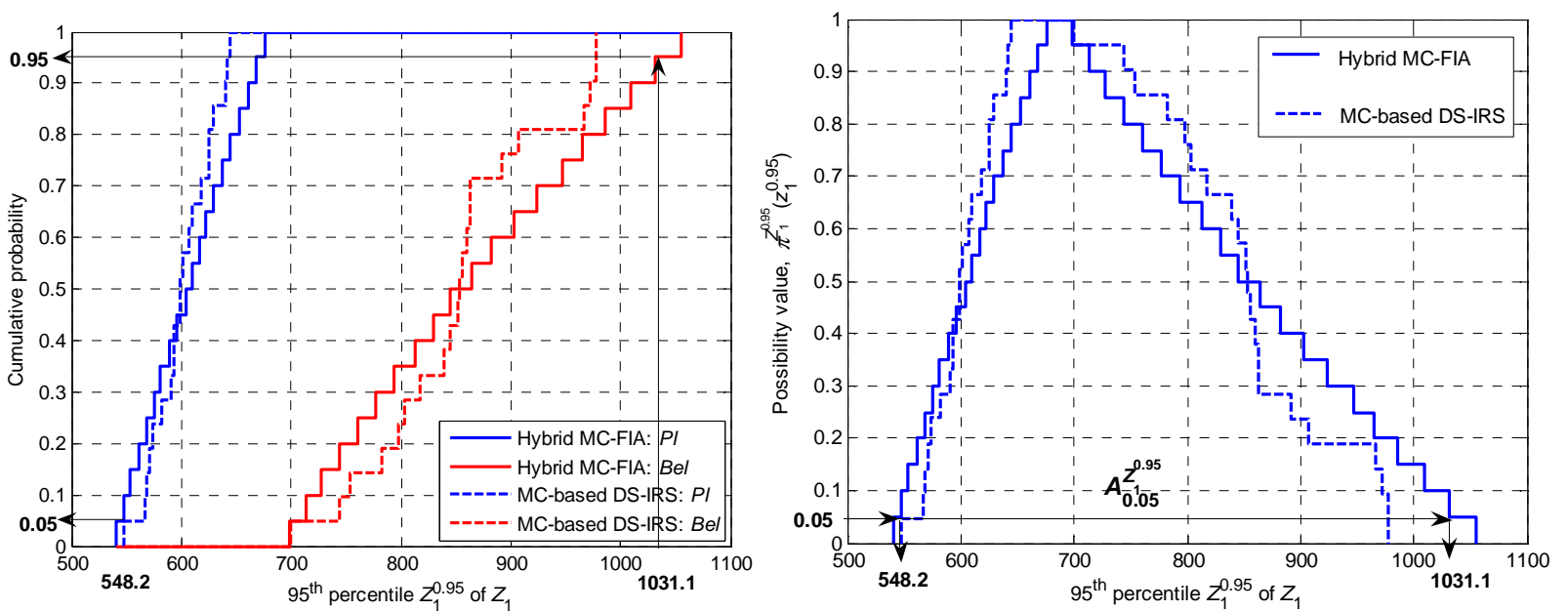

Figure 5. Left: upper and lower CDFs $\bar{F}^{Z_{1}^{0.95}}\left(z_{1}^{0.95}\right)$ and $\underline{F}^{Z_{1}^{0.95}}\left(z_{1}^{0.95}\right)$ of $Z_{1}^{0.95}$ obtained by the hybrid MC-FIA (solid lines) and MC-based DS-IRS (dashed lines) approaches. Right: possibility distributions $\pi^{Z_{1}^{0.95}}\left(z_{1}^{0.95}\right)$ corresponding to $\bar{F}^{Z_{1}^{0.95}}\left(z_{1}^{0.95}\right)$ and $\underline{F}^{Z_{1}^{0.95}}\left(z_{1}^{0.95}\right)^{(58)}$ 
It can be seen that the hybrid MC-FIA method produces a larger gap between the upper and lower CDFs $\bar{F}^{Z_{1}^{0.95}}\left(z_{1}^{0.95}\right)$ and $\underline{F}^{Z_{1}^{0.95}}\left(z_{1}^{0.95}\right)$ than the MC-based DS-IRS approach in the regions where the cumulative probabilities are close to "extreme" values, i.e., where $\bar{F}^{Z_{1}^{0.95}}\left(z_{1}^{0.95}\right) \approx 0$ and $\underline{F}^{Z_{1}^{0.95}}\left(z_{1}^{0.95}\right) \approx$ 1. This is explained as follows. Notice that the values of $Z_{1}^{0.95}$ for which $\bar{F}^{Z_{1}^{0.95}}\left(z_{1}^{0.95}\right) \approx 0$ and $\underline{F}^{Z_{1}^{0.95}}\left(z_{1}^{0.95}\right) \approx 1$ correspond to the lower and upper bounds, respectively, of the $\alpha$-cut of level $\alpha \approx 0$ of the possibility distribution $\pi^{Z_{1}^{0.95}}\left(z_{1}^{0.95}\right)$ (by way of example, the $\alpha$-cut $A_{0.05}^{Z_{1}^{0.95}}$ of level $\alpha=0.05$ produced by the hybrid MC-FIA is indicated by arrows in Figure 5, right). All this considered, it should be noticed that the $\alpha$-cut $A_{0.05}^{Z_{1}^{0.95}}$ of level $\alpha=0$ of the possibility distribution $\pi^{Z_{1}^{0.95}}\left(z_{1}^{0.95}\right)$ can be generated only by "combining" and propagating through the model function $Z_{1}=f_{Z l}\left(Y_{1}, Y_{2}, Y_{3}\right)$ the $\alpha$-cuts of level $\alpha=0$ of all the possibilistic parameters of the model inputs $Y_{1}, Y_{2}$ and $Y_{3}$. Such combination of $\alpha$-values, i.e., $\left\{\alpha_{1}=0, \alpha_{2}=0, \alpha_{3}=0\right\}$, is always "processed" by fuzzy interval analysis in the hybrid MC-FIA method, due to the underlying assumption of total dependence among the information sources (e.g., the experts or observers) that supply the parameters possibility distributions: actually, the same possibility (resp. confidence) level $\alpha$ (resp., $1-\alpha$ ) is chosen to build the $\alpha$-cuts for all the epistemically-uncertain parameters (see Section 4.2.1). On the contrary, such combination of possibility (resp., confidence) values, i.e., $\left\{\alpha_{1}=0, \alpha_{2}=0, \alpha_{3}=0\right\}$ (resp., $\left\{1-\alpha_{1}\right.$ $\left.=1,1-\alpha_{2}=1,1-\alpha_{3}=1\right\}$ ), cannot be obtained easily (i.e., with high probability) by the MC-based DS-IRS approach, which performs a plain random sampling among independent intervals. This is coherent with the real processes of expert elicitation, in that it is difficult to find different (independent) experts that provide estimates about different uncertain parameters with the same (and, in this case, maximal) confidence.

The higher conservatism of the hybrid MC-FIA approach is reflected, e.g., by the values of the exceedance probability $P\left[Z_{1}^{0.95}>z_{1}^{0.95^{*}}\right]$ (here $z_{1}^{0.95^{*}}=1000$ ). For example, it can be seen that $P\left[Z_{1}^{0.95}>z_{1}^{0.95^{*}}\right]$ ranges within $[0,0.1500]$ for the hybrid MC-FIA method, whereas it is 0 for the 
MC-based DS-IRS approach: thus, the uncertainty propagation performed by random sampling of independent focal sets leads to a dramatic underestimation of the exceedance probability ${ }^{(58)}$.

Some considerations are in order with respect to the results shown. The comparison shows that the choice of the uncertainty propagation method (and implicitly of the state of dependence among the epistemically-uncertain parameters of probability models) is not so critical (e.g., in risk-informed decisions) only when the extreme bounding upper and lower CDFs of the model output are of interest to the analysis: actually, the curves produced by the hybrid MC-FIA and the MC-based DSIRS approaches are almost identical. However, the analysis of other quantitative indicators (e.g., the distribution of a given quantile of the output) shows that the hybrid MC-FIA method produces a larger separation between the plausibility and belief functions than the MC-based DS-IRS approach, giving rise to more conservative results (in particular, in the range of small probabilities that are of particular interest in the risk assessment of complex, highly reliable systems).

\subsection{Updating the uncertainty representation with new information}

In all generality, let $\pi^{\theta}(\boldsymbol{\theta})$ be the (joint) prior possibility distribution for the epistemicallyuncertain parameters $\boldsymbol{\theta}=\left[\theta_{1}, \theta_{2}, \ldots, \theta_{m}, \ldots, \theta_{P}\right]$ of the (aleatory) PDF $p^{Y}(y \mid \boldsymbol{\theta})$ of input variable $Y$ (built on the basis of a priori subjective engineering knowledge and/or data). For example, in the flood risk assessment example used in what follows $Y$ may represent the yearly maximal water flow of a river described by the Gumbel distribution of Section 4.1: thus, $Y \sim p^{Y}(y \mid \boldsymbol{\theta})=G u m(\boldsymbol{\theta})=$ $\operatorname{Gum}(\gamma, \delta)=p^{Y}(y \mid \gamma, \delta)$ and $\pi^{\theta}(\boldsymbol{\theta})=\pi^{\gamma, \delta}(\gamma, \delta)$. Moreover, let $\boldsymbol{y}=\left[y_{1}, y_{2}, \ldots, y_{k}, \ldots, y_{D}\right]$ be a vector of $D$ observed pieces of data representing the new information/evidence available for the analysis: referring to the example above, $\boldsymbol{y}$ may represent a vector of $D$ values collected over a long period time (e.g., many years) of the yearly maximal water flow of the river under analysis. 
The new evidence acquired can be used to update the a priori uncertainty representation $\pi^{\theta}(\boldsymbol{\theta})=$ $\pi^{\gamma, \delta}(\gamma, \delta)$ of $\boldsymbol{\theta}=[\gamma, \delta]$, i.e., to calculate the posterior possibility distribution $\pi^{\theta}(\boldsymbol{\theta} \mid \boldsymbol{y})$ (i.e., $\left.\pi^{\gamma, \delta}(\gamma, \delta \mid \boldsymbol{y})\right)$ of $\boldsymbol{\theta}$ after $\boldsymbol{y}$ is obtained. In Section 4.3.1, a method based on a purely possibilistic counterpart of the classical, probabilistic Bayes theorem is suggested for the updating; in Section 4.3.2, the effectiveness of the recommended approach is assessed, for the first time to the best of the authors' knowledge, by comparison with a hybrid probabilistic-possibilistic technique of literature.

\subsubsection{Recommended approach: purely possibilistic Bayes' theorem}

The purely possibilistic method (hereafter also referred to as 'Approach A' for brevity) is based on a purely possibilistic counterpart of the classical, probabilistic Bayes theorem ${ }^{(112,113)}$ :

$$
\pi^{\theta}(\boldsymbol{\theta} \mid \boldsymbol{y})=\frac{\pi_{L}^{\theta}(\boldsymbol{\theta} \mid \boldsymbol{y}) \cdot \pi^{\theta}(\boldsymbol{\theta})}{\sup _{\boldsymbol{\theta}}\left\{\pi_{L}^{\theta}(\boldsymbol{\theta} \mid \boldsymbol{y}) \cdot \pi^{\theta}(\boldsymbol{\theta})\right\}}
$$

where $\pi_{L}^{\theta}(\boldsymbol{\theta} \mid \boldsymbol{y})$ is the possibilistic likelihood of the parameter vector $\boldsymbol{\theta}$ given the newly observed data $\boldsymbol{y}$, and quantities $\pi^{\theta}(\boldsymbol{\theta} \mid \boldsymbol{y})$ and $\pi^{\theta}(\boldsymbol{\theta})$ are defined above. Notice that $\sup _{\boldsymbol{\theta}}\left\{\pi_{L}^{\theta}(\boldsymbol{\theta} \mid \boldsymbol{y}) \cdot \pi^{\boldsymbol{\theta}}(\boldsymbol{\theta})\right\}$ is a normalization factor such that $\sup _{\boldsymbol{\theta}}\left\{\pi^{\theta}(\boldsymbol{\theta} \mid \boldsymbol{y})\right\}=1$, as required by possibility theory ${ }^{(50)}$.

It is worth mentioning that forms of the possibilistic Bayes theorem alternative to (2) can be constructed as a result of other definitions of the operation of 'conditioning' with possibility distributions: the reader is referred to Refs. 112 and 113 for technical details. In this paper, expression (2) is recommended because "it satisfies desirable properties of the revision process and leads to continuous posterior distributions"(113).

The possibilistic likelihood $\pi_{L}^{\theta}(\boldsymbol{\theta} \mid \boldsymbol{y})$ is here obtained by transforming the classical probabilistic likelihood function $L^{\theta}(\boldsymbol{\theta} \mid \boldsymbol{y})$ through normalization, i.e., $\pi_{L}^{\theta}(\boldsymbol{\theta} \mid \boldsymbol{y})=\frac{L^{\theta}(\boldsymbol{\theta} \mid \boldsymbol{y})}{\sup _{\boldsymbol{\theta}}\left\{L^{\theta}(\boldsymbol{\theta} \mid \boldsymbol{y})\right\}}{ }^{(137)}$. This choice has been made for the following main reasons: 
i. $\quad$ the transformation is simple and can be straightforwardly applied to any distribution ${ }^{(137)}$;

ii. the resulting possibilistic likelihood is closely related to the classical, purely probabilistic one (which is theoretically well-grounded) by means of the simple and direct operation of normalization that preserves the "original structure" of the experimental evidence;

iii. it can be easily verified that the resulting possibilistic likelihood keeps the sequential nature of the updating procedure typical of the standard Bayes theorem.

Notice that other techniques of transformation of probability density functions into possibility distributions exist: see Refs. 132, 134 and 135. Techniques are also available to construct possibility distributions (and, thus, possibilistic likelihood functions) directly from experimental data (i.e., without resorting to "artificial" transformations): see Refs. 138 and 139 for details.

\subsubsection{Illustrative example}

For completeness, we report (and extend) some of the results obtained in a previous work by the authors $^{(61)}$, in which the purely possibilistic Bayes' theorem described above is applied for updating the possibilistic parameters of aleatory PDFs in a simple literature case study involving the riskbased design of a flood protection dike ${ }^{(62)}$. In the risk model considered the maximal water level $Z_{c}$ of a river (i.e., the output variable $Z$ of the model) is given as a function of several (uncertain) parameters (the inputs $\boldsymbol{Y}$ to the model), i.e., $Z=f_{Z}\left(Y_{1}, Y_{2}, Y_{3}, Y_{4}\right)=Z_{c}=f_{Z_{c}}\left(Q, Z_{m}, Z_{v}, K_{s}\right)$, with $Y_{1}=$ $Q \sim \operatorname{Gum}(\gamma, \delta), Y_{2}=Z_{m} \sim N\left(\mu_{Z m}, \sigma_{Z m}\right), Y_{3}=Z_{v} \sim N\left(\mu_{Z v}, \sigma_{Z v}\right)$ and $Y_{4}=K_{s} \sim N\left(\mu_{K s}, \sigma_{K s}\right)$; the 'priors' of the parameters of the aleatory PDFs of the inputs are represented by triangular possibility distributions: see Ref. 61 for further details.

The benefits coming from the use of the proposed method are here shown by means of a comparison to the hybrid probabilistic-possibilistic approach proposed in Ref. 114 (hereafter also called 'Approach B' for brevity) and based on the combination of: (i) FIA to process the uncertainty 
described by possibility distributions; and (ii) repeated Bayesian updating of the uncertainty represented by probability distributions.

In order to simplify the notation, in what follows let $\theta$ be one of the uncertain parameters of the PDFs of $Y_{1}=Q, Y_{2}=Z_{m}, Y_{3}=Z_{v}$ and $Y_{4}=K_{s}$, i.e., $\theta=\gamma, \delta, \mu_{Z m}, \sigma_{Z m}, \mu_{Z v}, \sigma_{Z v}, \mu_{K_{s}}$ or $\sigma_{K s}$. By way of example, Figure 6 illustrates the possibility distributions of the epistemically-uncertain parameters $\gamma$ and $\sigma_{K s}$ of the aleatory PDFs $p^{Q}(q \mid \gamma, \delta)$ (left) and $p^{K_{s}}\left(k_{s} \mid \mu_{K s}, \sigma_{K s}\right)$ (right) of $Y_{1}=Q$ and $Y_{4}=K_{s}$, respectively: in particular, the prior possibility distributions $\pi^{\theta}(\theta)$ are shown as solid lines, whereas the marginal posterior possibility distributions $\pi^{\theta}(\theta \mid \boldsymbol{y})$ obtained by Approaches A (Section 4.3.1) and $\mathrm{B}^{(114)}$ using $D_{1}=149$ and $D_{4}=5$ pieces of data are shown in dashed and dotdashed lines, respectively; the point estimates $\hat{\theta}^{M L E}$ produced by the classical MLE method are also shown for comparison $(\operatorname{dots})^{(61,62)}$.
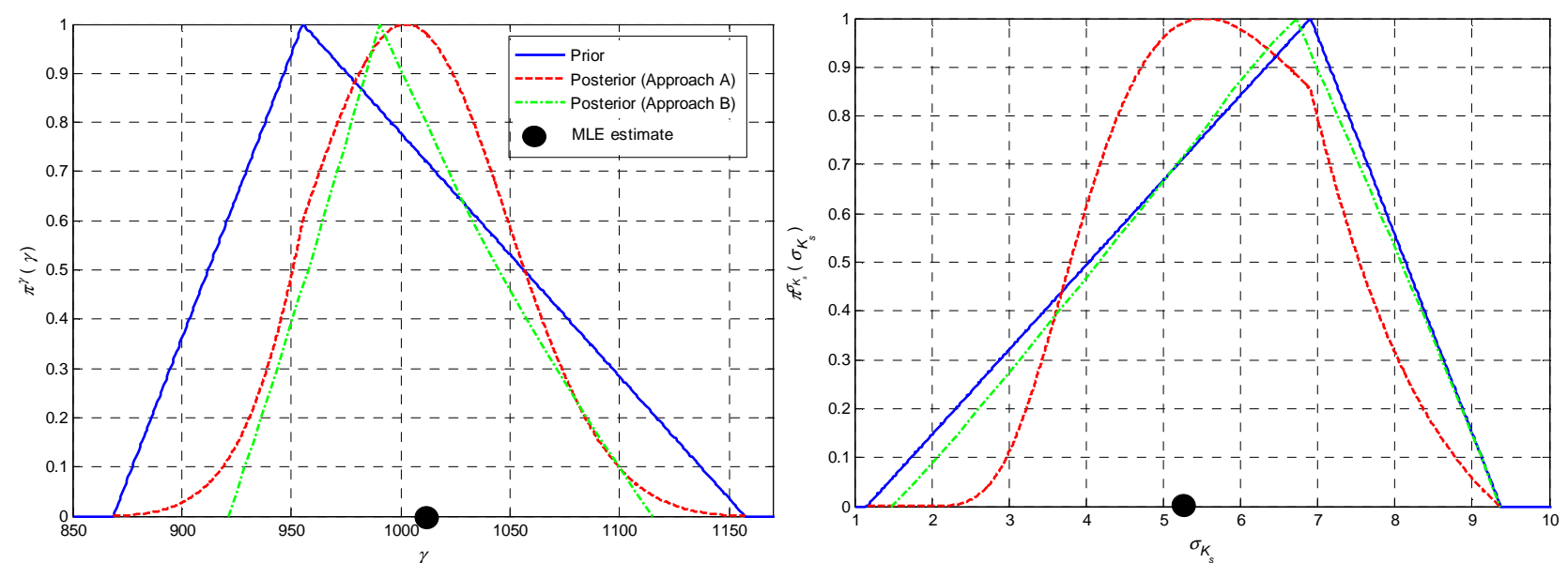

Figure 6. Prior and posterior possibility distributions of the epistemically-uncertain parameters $\gamma$ and $\sigma_{K s}$ of the aleatory PDFs $p^{Q}(q \mid \gamma, \delta)$ (left) and $p^{K_{s}}\left(k_{s} \mid \mu_{K s}, \sigma_{K s}\right)$ (right) of $Y_{1}=Q$ and $Y_{4}=K_{s .}$.

The point estimated obtained by the classical MLE method are shown for comparison

From a mere visual and qualitative inspection of Figure 6, it can be seen that both approaches are suitable for revising the prior possibility distributions by means of empirical data. In particular, it is evident that: (i) the most likely (i.e., preferred) values $c_{\theta}$ of the epistemically-uncertain parameters (i.e., those values in correspondence of which the possibility function equals 1) are moved towards 
the MLE estimates $\hat{\theta}^{M L E}$ in all the cases considered; (ii) the area $S_{\theta}$ underlying the corresponding possibility distributions is significantly reduced: noting that this area is related to the imprecision in the knowledge of the possibilistic parameter (i.e., the larger the area, the higher the imprecision), it can be concluded that both approaches succeed in reducing the epistemic uncertainty (nevertheless, note that the agreement between the results obtained with the two numerical procedures does not necessarily establish the correctness or appropriateness of those procedures).

On the other hand, it is evident that the strength of Approach A in moving the most likely values $c_{\theta}$ towards the corresponding MLE estimates $\hat{\theta}^{M L E}$ is always higher than that of Approach B. Actually, the distances between the MLE estimates $\hat{\theta}^{M L E}$ and the posterior most likely values produced by Approach A are 1.35-7.94 times lower than those generated by Approach B.

In addition, it is interesting to note that the strength of Approach B in reducing epistemic uncertainty is slightly higher than that of Approach A only when the amount of available data is quite large (i.e., in the revision of the possibility distributions of parameters $\gamma$ and $\delta$ of the PDF of $Y_{1}=Q$, by means of $D_{1}=149$ pieces of data): actually, the areas underlying the corresponding possibility distributions are reduced by $25.56-30.49 \%$ and $28.74-33.01 \%$ by Approaches A and B, respectively. In all the other cases, the power of Approach A in reducing the epistemic uncertainty is higher than that of Approach B and this difference becomes more and more evident as the size of the data set decreases. This is particularly evident in the estimation of the standard deviation $\sigma_{K s}$ of $K_{s}$ (Figure 6, right): on one side, the posterior distribution produced by the hybrid approach (B) seems not to be influenced by the revision process (actually, the most likely value of the parameter, $c_{\sigma_{K s}}=6.72$, and the area underlying the corresponding posterior possibility distribution, $S_{\sigma_{K s}}=$ 3.95, are quite close to those of the prior, i.e., 6.89 and 4.11, respectively); on the other side, the posterior distribution generated by the purely possibilistic approach (A) is almost centered on the point estimates obtained by the MLE method and the corresponding area is reduced by about $9 \%$. 
Finally, in addition to the strength of the approaches in revising the (prior) possibilistic description of the uncertain parameters of aleatory variables, also the computational cost associated to the methods has to be taken into account. In this respect, the time $t_{c o m p}$ required by Approach $\mathrm{B}$ is approximately $T \cdot N_{\alpha}$ times larger than that of Approach A, since it entails $T$ repetitions of the purely probabilistic Bayes theorem for each of the $N_{\alpha} \alpha$-cuts analyzed (in this case, $T \cdot N_{\alpha}=100 \cdot 21=2100$ ): see Ref. 114 for details.

Several considerations are in order with respect to the results obtained. Both methods succeed in updating the possibilistic description of the epistemically-uncertain parameters of (aleatory) probability distributions by means of data. In addition, when the Bayesian update is performed based on a data set of large size (e.g., > 100 in this case), the strength of the two approaches in reducing the epistemic uncertainty is quite similar. This demonstrates that although the two methods are conceptually and algorithmically quite different, in presence of a "strong" experimental evidence they produce "coherent" results (i.e., posterior possibility distributions that bear the same overall "uncertainty content"): this is a fair outcome since the results provided by the two methods are expected to be more and more similar (i.e., more and more coherent with the experimental evidence) as the size of the data set increases. Instead, the strength of the purely possibilistic approach (A) in reducing epistemic uncertainty is consistently higher than that of the hybrid one (B) in presence of medium- and small-sized data sets (e.g., $\approx 5-30$ in the present study) (which is often the case in the risk analysis of complex safety-critical systems). In such cases, embracing one method instead of the other may significantly change the outcome of a decision making process in a risk assessment problem involving uncertainties: this is of paramount importance in systems that are critical from the safety view point, e.g., in the nuclear, aerospace, chemical and environmental fields.

However, it is absolutely important to acknowledge that even if the strength of method A in reducing epistemic uncertainty is higher than that of method $\mathrm{B}$, this does not necessarily imply that 
method A is "better" or "more effective" than method B overall. Actually, if on one side a consistent reduction in the epistemic uncertainty is in general desirable in decision making processes related to risk assessment problems (since it significantly increases the analyst confidence in the decisions), on the other side this reduction must be coherent with the amount of information available. In this view, an objection may arise in the present case: is the remarkable strength of Approach A in reducing epistemic uncertainty (with very few pieces of data) fully justified by such a small amount of data? In other words, is this considerable reduction of epistemic uncertainty coherent with the strength of the experimental evidence or is it too optimistic? With respect to that, it has to be admitted that the uncertainty reduction power of the purely possibilistic approach (A) is strongly dependent on the shape of an artificially constructed possibilistic likelihood that could in principle bias the analysis. However: (i) in the approach recommended in the present paper, this possibilistic function is very closely related to the classical, purely probabilistic one (which is theoretically well-grounded) by a simple and direct operation of normalization that preserves the "original structure" of the experimental evidence; (ii) in general, a probability-to-possibility transformation (properly performed according to the rules of possibility theory) always introduces additional artificial epistemic uncertainty into the analysis, i.e., it does not artificially reduce it (because it replaces a single probabilistic distribution by a family of distributions) $)^{(132,134,135)}$. On the basis of the considerations above, it seems unlikely that the purely possibilistic approach (A) may produce results that are dangerously over-optimistic with respect to those of the hybrid one (B).

Finally, the computational time required by the hybrid approach (B) is consistently (i.e., hundreds or thousands times) higher than that associated to the purely probabilistic one (A): this is explained by the necessity of repeatedly applying many (e.g., hundreds) times the purely probabilistic Bayes' theorem for each $\alpha$-cut analyzed. 


\subsection{Dependence among the input variables and parameters}

As discussed in Section 3.4, both objective and state-of-knowledge dependences need to be considered in risk assessment analyses ${ }^{(7,33,34)}$. However, in many practical cases the state of dependence among the uncertain model parameters and variables is difficult to define precisely. In such situations, conservatism requires that all kinds of (possibly unknown) dependences be accounted for. To do this, the Distribution Envelope Determination (DEnv) method is here recommended $^{(126-129)}$. In Section 4.4.1 the method is outlined in detail; a demonstration of the approach on a case study concerning Fault Tree Analysis (FTA) is given in Section 4.4.2.

\subsubsection{Recommended approach: Distribution Envelope Determination (DEnv) method}

The DEnv method allows computing extreme upper and lower CDFs $\bar{F}_{D E n v}^{Z}(z)$ and $\underline{F}_{D E n v}^{Z}(z)$ on the output $Z$ of the model $f_{Z}\left(Y_{1}, Y_{2}, \ldots, Y_{j}, \ldots, Y_{N}\right)$ (1) no matter what dependencies exist among the inputs; these bounds are also the "pointwise best possible, which means they could not be any tighter without excluding some possible dependences"(33). Notice that this approach can be applied both at the objective and epistemic levels ${ }^{(58,59)}$. The method requires the following steps ${ }^{(126-129)}$ :

1. represent the uncertainty on the inputs $Y_{1}, Y_{2}, \ldots, Y_{j}, \ldots, Y_{N}$ within the framework of Dempster-Shafer (DS) theory of evidence. The application of evidence theory produces a description of the inputs in terms of so-called DS structures $\left\{\left(A_{Y_{j}}^{i_{j}}, m\left(A_{Y_{j}}^{i_{j}}\right)\right): i_{j}=1,2, \ldots, n_{j}\right\}, j=$ $1,2, \ldots, N$ : in other words, input $Y_{j}$ is described by a set of $n_{j}$ intervals (focal elements) $A_{Y_{j}}^{i_{j}}=\left[\underline{y}_{j}^{i_{j}}, \bar{y}_{j}^{i_{j}}\right]$ each of which is assigned a probability (or belief) mass $m\left(A_{Y_{j}}^{i_{j}}\right), i_{j}=1,2, \ldots$, $n_{j}, j=1,2, \ldots, N$. Notice that the DS structures described above can be transformed into upper and lower Cumulative Distribution Functions (CDFs) $\bar{F}^{Y_{1}}$ and $\underline{F}^{Y_{1}}$ (called cumulative plausibility and belief functions, respectively): in particular, $\bar{F}^{Y_{1}}\left(y_{1}\right)=\bar{P}\left[Y_{1}<y_{1}\right]=$ 
$\sum_{A_{Y_{1}}^{i_{1}} \cap\left[0, y_{1}\right] \neq 0} m\left(A_{Y_{1}}^{i_{1}}\right)$ and $\underline{F}^{Y_{1}}\left(y_{1}\right)=\underline{P}\left[Y_{1}<y_{1}\right]=\sum_{A_{Y_{1}}^{i_{1}} \in\left[0, y_{1}\right]} m\left(A_{Y_{1}}^{i_{1}}\right)$. An exemplary DS structure and the corresponding upper and lower CDFs are pictorially shown in Figure $7^{\mathrm{j}}(33,43,44,46,85)$;
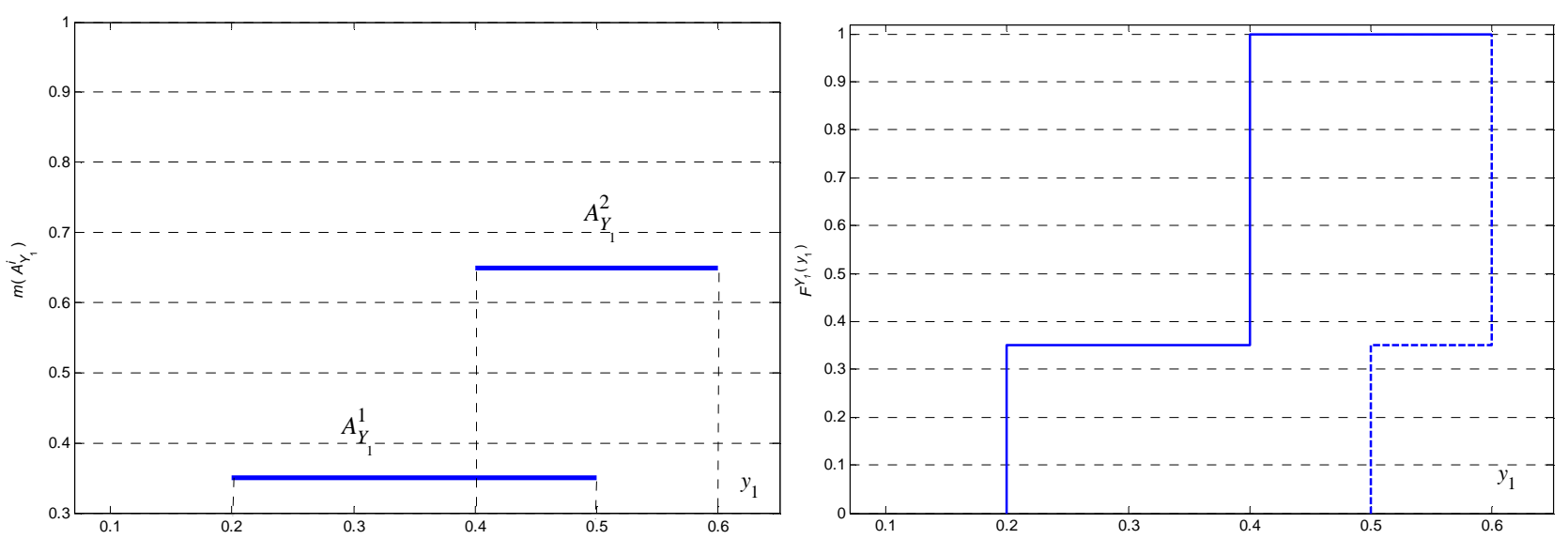

Figure 7. Exemplary DS structure (left) and corresponding upper and lower CDFs (right)

2. propagate the input focal elements through the model $f_{Z}\left(Y_{1}, Y_{2}, \ldots, Y_{N}\right)$ to obtain the output focal elements $A_{Z}^{i_{1} i_{2} \ldots i_{j} \ldots i_{N}}=\left[\underline{z}^{i_{i} i_{2} \ldots i_{j} \ldots i_{N}}, \bar{z}^{i_{1} i_{2} \ldots i_{j} \ldots i_{N}}\right]$ as $\left[\min _{Y_{j} \in A_{Y_{j}}^{i_{j}}: j=1,2, \ldots, N}\left\{f_{Z}\left(Y_{1}, Y_{2}, \ldots, Y_{j}, \ldots, Y_{N}\right)\right\}\right.$, $\left.\max _{Y_{j} \in A_{Y_{j}}^{i_{j}}: j=1,2, \ldots, N}\left\{f_{Z}\left(Y_{1}, Y_{2}, \ldots, Y_{j}, \ldots, Y_{N}\right)\right\}\right], i_{j}=1,2, \ldots, n_{j}, j=1,2, \ldots, N$

3. properly assign the (joint) probability masses $m\left(A_{Z}^{i_{2} i_{2} \ldots i_{j} \ldots i_{N}}\right)$ to the output focal elements $A_{Z}^{i_{i} i_{2} \ldots i_{j} \ldots i_{N}}$ obtained at step 2. above in such a way that the resulting upper CDF on $Z$ is the maximal possible (i.e., $\bar{F}_{D E n v}^{Z}(z)=\max \left\{\bar{F}^{Z}(z)\right\}$ ) and the resulting lower $\operatorname{CDF}$ on $Z$ is the minimal possible $\left(\underline{F}_{D E n v}^{Z}(z)=\min \left\{\underline{F}^{Z}(z)\right\}\right)$ given a precise set of constraints ${ }^{(126-129)}$ :

Find $m\left(A_{Z}^{i_{\mathrm{i}} i_{2} \ldots i_{j} \ldots i_{N}}\right), i_{j}=1,2, \ldots, n_{j}, j=1,2, \ldots, N$ :

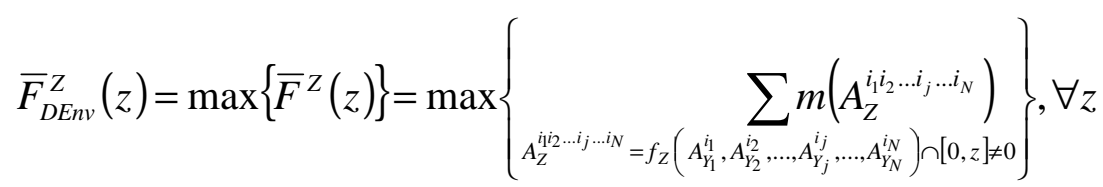

\footnotetext{
${ }^{\mathrm{j}}$ Notice that representing the uncertainty in the inputs $Y_{1}, Y_{2}, \ldots, Y_{j}, \ldots, Y_{N}$ by DS structures does not impair the generality of the description. Actually, any other type of distribution that may be used to describe the uncertainty in $Y_{1}$, $Y_{2}, \ldots, Y_{j}, \ldots, Y_{N}$ can be easily transformed into a DS structure: approaches for transforming probability distributions can be found in Ref. 102, whereas techniques for transforming possibility distributions can be found in Ref. 33 .
} 
Find $m\left(A_{Z}^{i_{1} i_{2} \ldots i_{j} \ldots i_{N}}\right), i_{j}=1,2, \ldots, n_{j}, j=1,2, \ldots, N$ :

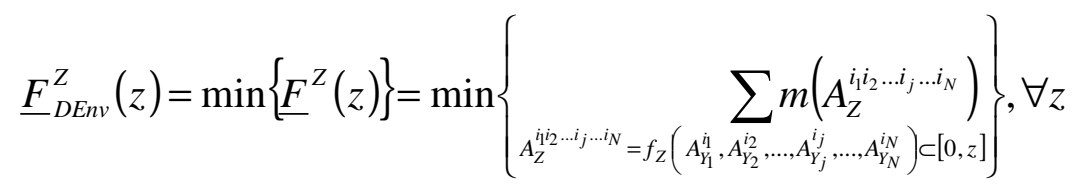

subject to the constraints that: (i) the probability masses $m\left(A_{Y_{j}}^{i_{j}}\right)$ are conserved (i.e., $\left.\sum_{i_{1}=1}^{n_{1}} \sum_{i_{2}=1}^{n_{2}} \ldots \sum_{i_{j-1}=1}^{n_{j-1}} \sum_{i_{j+1}=1}^{n_{j+1}} \ldots \sum_{i_{N}=1}^{n_{N}} m\left(A_{Z}^{i_{1} i_{2} \ldots i_{j} \ldots i_{N}}\right)=m\left(A_{Y_{j}}^{i_{j}}\right), i_{j}=1,2, \ldots, n_{j}, j=1,2, \ldots, N\right)$; and (ii) the probability masses $m\left(A_{Z}^{i_{2} i_{2} \ldots i_{j} \ldots i_{N}}\right)$ are larger than or equal to zero.

It is worth noting that in order to construct the entire CDFs $\bar{F}_{D E n v}^{Z}(z)$ and $\underline{F}_{D E n v}^{Z}(z)$ for $Z$, such optimization problems have to be solved for all the values $z$ of interest.

Finally, notice that an alternative sampling-based approach to (i) the propagation of a DS structure through a model and (ii) the construction of approximations to the cumulative plausibility and belief functions can be found in Refs. 39 and 44; however, being a sampling-based strategy, this approach cannot encompass the treatment of unknown dependences between uncertain variables.

\subsubsection{Illustrative example}

In what follows, we report some of the results obtained in a previous work by some of the authors $^{(59)}$, in which the effects of objective and epistemic dependences are analyzed with reference to the Top Event (TE) probability $P(X)$ of a Fault Tree (FT) containing $n_{B E}=6$ Basic Events (BEs) with epistemically-uncertain probabilities $\left\{P\left(B_{j}\right): j=1,2, \ldots, n_{B E}=6\right\}$. The order of magnitude of the BE probabilities is around $10^{-3}$ : this is reasonable for realistic safety-critical systems where the components are usually highly reliable. Further details can be found in the cited reference.

Two classes of analyses are performed to analyze the effects of different states of objective (Analysis 1) and epistemic (Analysis 2) dependence among the BEs. In Analysis 1, three configurations (namely, T1-T3) are considered: Configuration T1 represents the reference, baseline case where all the BEs are considered (objectively) independent. On the opposite, Configuration T3 
represents the extreme (most conservative) case where no assumptions about the states of objective dependence among all the BEs are made. Instead, Configuration T2 represents an 'intermediate' case. In particular, in Configuration T2 positive objective dependence is assumed between two of the six BEs (in this case, between two events representing failures of mechanical components): this situation is far from unlikely in real systems and may be due to several causes, e.g., (i) shared pieces of equipment (e.g., components in different systems are fed from the same electrical bus) or (ii) physical interactions (e.g., failures of some component create extreme environmental stresses, which increase the probability of multiple-component failures). Finally, in Analysis 2 only three 'extreme' situations (namely, E1-E3) are considered: in particular, in Configurations E1, E2 and E3 states of independence, total (perfect) dependence and unknown epistemic dependence, respectively, are assumed among all the probabilities of all the BEs of the FT.

Figure 8, left, depicts the upper and lower CDFs $\bar{F}^{P(X)}$ and $\underline{F}^{P(X)}$ obtained for $P(X)$ under different assumptions of objective dependence among the BEs (Analysis 1). In order to provide a quantitative evaluation of the effects of such states of objective dependence, the interval $\left[\underline{p}_{X}^{0.95}, \bar{p}_{X}^{0.95}\right]=$ $\left[\left(\bar{F}^{P(X)}\right)^{-1}(0.95),\left(\underline{F}^{P(X)}\right)^{-1}(0.95)\right]$ for the 95 -th percentile $P(X)^{0.95}$ of $P(X)$ is computed. Notice that $\bar{p}_{X}^{0.95}=\left(\underline{F}^{P(X)}\right)^{-1}(0.95)$ can be interpreted as a conservative assignment of $P(X)^{0.95}$ (i.e., a conservative estimate of risk). It can be seen that the values of $\bar{p}_{X}^{0.95}$ are $7.23 \cdot 10^{-4}$ and $8.98 \cdot 10^{-3}$ in Configurations $\mathrm{T} 1$ and $\mathrm{T} 2$. This means that neglecting a hypothetical state of positive dependence between only one pair of BEs is sufficient for underestimating $\bar{p}_{X}^{0.95}$ (and, thus, the risk associated to the system) by 12.42 times. Finally, Configuration T3 represents the 'extreme' case where unknown objective dependence is assumed among all the BEs of the FT. Actually, the value of $\bar{p}_{X}^{0.95}$ is $2.59 \cdot 10^{-2}$, i.e., 35.87 times larger than those obtained under the 'baseline' assumption of objective independence among all the BEs (Configuration T1). 
With reference instead to Analysis 2, Figure 8, right, depicts the upper and lower CDFs $\bar{F}^{P(X)}$ and $\underline{F}^{P(X)}$ obtained under different states of epistemic dependence among the probabilities of all the BEs (Analysis 2). It is evident that the upper and lower CDFs $\bar{F}^{P(X)}$ and $\underline{F}^{P(X)}$ obtained under the assumption of unknown epistemic dependence (dot-dashed lines) completely envelop all the others (i.e., they obviously represent more conservative estimates of the bounding distributions). In addition, it is worth noting that the lower (resp., upper) CDFs obtained under the assumption of perfect epistemic dependence are very close to those produced by the assumption of unknown epistemic dependence in the region where the cumulative probability is very close to the 'extreme' upper bound 1 (resp., lower bound 0). In other words, the CDFs produced under assumptions of perfect and unknown epistemic dependence are almost identical in the range of extreme probabilities (i.e., extreme quantiles) that are of particular interest in the risk assessment of highly reliable systems. This is confirmed by the analysis of $\bar{p}_{X}^{0.95}$, whose values are $4.40 \cdot 10^{-4}, 6.41 \cdot 10^{-4}$ and $7.23 \cdot 10^{-4}$ under the assumptions of independence, total and unknown dependence, respectively.
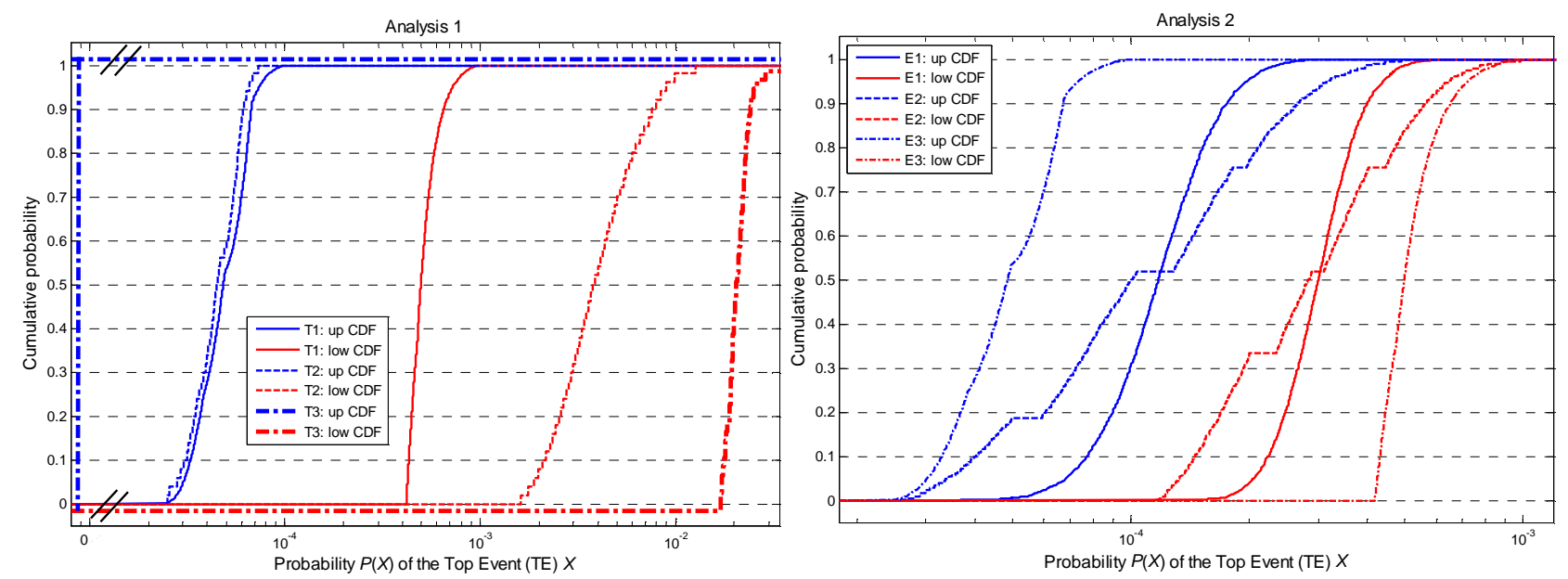

Figure 8. Effect of objective (left) and epistemic (right) dependences on $P(X)$

Some considerations are in order with respect to the results obtained. With respect to Analysis 1, the assumption of objective independence among the BEs always leads to a serious underestimation of the risk associated to the system (here represented by the upper bound of the 95-th quantile of the TE probability) with respect to the assumptions of perfect and unknown objective dependence: 
actually, the corresponding estimates may differ even by orders of magnitude. Moreover, this underestimation is shown to be quite dramatic for small BE probabilities (e.g., around $10^{-3}$ like in the present case): this poses concerns for the risk assessment of systems where the components are highly reliable and, thus, characterized by small failure probabilities.

With respect to Analysis 2, it is shown that: (i) the assumption of epistemic independence among the probabilities of random events leads to a non-negligible underestimation of the risk associated to the system (here represented by the upper bound of the 95-th quantile of the TE probability) with respect to the assumptions of perfect and unknown epistemic dependence (e.g., by about 1.5 times): this is particularly evident in the estimation of small probabilities and extreme quantiles that are of paramount importance in the risk assessment of highly reliable systems; (ii) the estimates for the upper bound of the 95-th quantile of the TE probability produced by the assumptions of perfect and unknown epistemic dependence are comparable and (iii) the effects of epistemic dependence among the BE probabilities are quantitatively less relevant and critical than those of objective dependence among the BEs.

\section{CONCLUSIONS AND DISCUSSION}

In this paper, the following conceptual and technical issues on the uncertainty treatment in the risk assessment of engineering systems have been considered: (1) quantitative modeling and representation of uncertainty, coherently with the information available on the system of interest; (2) propagation of the uncertainty from the input(s) to the output(s) of the model of the system; (3) (Bayesian) updating of the uncertainty representation as new information becomes available; (4) modeling and representation of dependences among the model input variables and parameters. Different approaches to tackle each of the issues 1.-4. listed, outside a fully probabilistic framework have been compared. On the basis of the comparisons and of previous research by the authors $^{(58-61)}$, the following guidelines and recommendations have been drawn: 
1. for the first issue, the Fuzzy Random Variable (FRV) approach can be one of those recommended for uncertainty modeling and representation, in particular when the data and information available on the problem of interest are scarce, vague and/or imprecise. In such a framework, aleatory uncertainty is represented by probability models (i.e., probability distributions), whereas epistemic uncertainty in the internal parameters of the aleatory models is described by possibility distributions. The resulting FRV defines a family of nested pairs of aleatory probability distributions, each of which bounds the "true" probability distribution with a given confidence level. In the examples here proposed, the FRV approach has been shown to provide more conservative results than the classical, purely probabilistic one in the estimation of important quantities, like the distribution of a quantile of the model output. On the other hand, this does not mean that possibility distributions should be always used to represent epistemic uncertainty. Actually: (i) other non-probabilistic approaches exist for tackling problems characterized by imprecise information (see, e.g., evidence theory); (ii) in some cases (e.g., in the presence of a relevant amount of data) also classical probability theory can obviously serve this purpose;

2. for the second issue, in general the hierarchical propagation of hybrid aleatory (probabilistic) and epistemic (possibilistic) uncertainty should be carried out coherently with the state of dependence between the epistemically-uncertain parameters, if known. On the other hand, if the objective of the analyst is that of producing conservative risk estimates, then the MCFIA approach should be adopted. Actually, it has been show to provide more conservative results than the MC-based DS-IRS approach in the estimation of the distributions of a given quantile of the model output. In addition, this higher conservatism is particularly evident in the range of extreme probabilities (i.e., around 0 and 1) that are of paramount importance in realistic risk assessment applications involving highly reliable engineering systems; 
3. for the third issue, the purely possibilistic counterpart of the classical probabilistic Bayes' theorem is strongly suggested (instead of the hybrid one) for updating, in a Bayesian framework, the possibilistic representation of the epistemically-uncertain parameters of (aleatory) probability distributions. This is due to the following reasons: (i) its strength in reducing epistemic uncertainty is significantly higher, in particular when the amount of available data is small: this is important in decision making processes since reducing epistemic uncertainty significantly increases the analyst confidence in the decisions; (ii) the computational time required is consistently lower. However, it has to be remarked that the construction of a possibilistic likelihood required by this method still represents an issue to be tackled from the theoretical and practical viewpoint in order to avoid introducing biases in the analysis: with respect to that, future research will be devoted to the investigation of additional methods, either resorting to probability-possibility transformations or (preferably) building possibilistic functions directly from rough experimental data;

4. for the fourth issue, it has been shown that (i) the treatment of objective dependences among random events is very critical since they have a dramatic impact on the system risk measures: in this view, unknown (or, at least, positive) objective dependence should be assumed among random events, in particular if the corresponding probabilities are small (e.g., of the order of $10^{-3}-10^{-2}$ ); (ii) the conditions of epistemic dependence should not be neglected when small probabilities and extreme quantiles have to be estimated: with respect to that, unknown (or, at least, perfect) epistemic dependences should be assumed in order to obtain conservative risk estimates; however, if objective dependences are also present, the effects of epistemic dependence are likely to be overwhelmed by those of objective dependence. In the light of these results, the DEnv method is strongly recommended to account for all kinds of (possibly unknown) objective and epistemic dependences. 
Tackling these issues is undoubtedly a step forward towards the use of non-probabilistic settings for risk analysis in engineering and gives encouraging perspectives for future studies. Without taking any dogmatic position, the proposed methodology could be considered as an alternative to the more common probabilistic approach.

However, these conclusions should not hide some difficulties in the practical use of these methods which are currently partially addressed and should be more investigated in future works. A first limitation comes from the computational cost of these approaches, which can become prohibitive when the system model is a CPU time consuming code as it can be often the case in complex system safety studies (e.g. finite elements CFD or mechanical codes, multi-physical coupling of models). This problem can be tackled by distributed computing and/or meta-modeling techniques (the latters adding an additional level of uncertainty).

Another interesting perspective for future work is the definition of point risk indicators (e.g. a probability of failure). That can be done quite easily (at least by a conceptual viewpoint) in the fully-probabilistic framework: the Bayesian point estimator is the value minimizing the expectation of a loss function depending on the "distance" of the proposed value from the real (albeit unknown) one, the loss function encoding the conservatism one wants to give to the point risk indicator ${ }^{(107)}$. In some studies, it should be interesting to have at disposal a formal theory for choosing a point value of the quantity of interest to be estimated, especially when the interval resulting from the methodology shown hereinbefore is very large. Depending on the context, the counterpart of the conservatism of these intervals is the difficulty in making decision under their base.

As a closing general comment, it is worth pointing out that although in some of the recommendations above we have chosen "informed" conservatism as a possible criterion of comparison between the probabilistic and possibilistic methods, we do not want to overstate the benefits of conservatism in itself. Actually, (i) assumptions that are conservative without justification may cause a misallocation of resources; (ii) excessive conservatism can impair the 
results of an entire analysis, because it makes the analysts appear to lack an understanding of the problem under consideration; (iii) in some cases, an assumption, which is conservative with respect to some results of a given analysis, may be non-conservative with respect to other results of the same analysis. Overly conservative assumptions can be as damaging to decision making as overly optimistic assumptions (i.e., not "anchored" to the available information). In an analysis performed to support a possibly critical, safety-related decision, the appropriate goal is to be neither overly optimistic nor overly pessimistic in the assumptions used, but rather to use "objective" and rigorous tools for transforming the available information into a "faithful" description of the uncertainties that are present in the analysis and its results.

\section{REFERENCES}

1. Aven T. Risk assessment and risk management: Review of recent advances on their foundation. European Journal of Operational Research 2016; 253(1): 1-13.

2. SRA (Society of Risk Analysis). Glossary of the specialty group on Foundations of Risk Analysis. http://www.sra.org/news/sra-develops-glossary-risk-related-terms; 2015.

3. Apostolakis GE. The concept of probability in safety assessment of technological systems. Science 1990; 250: 1359-1364.

4. NASA (National Aeronautics and Space Administration). Risk-Informed Decision Making Handbook. NASA/SP-2010-576 - Version 1.0. Washington, DC (USA): Office of Safety and Mission Assurance, NASA Headquarters; April 2010.

5. USNRC. Reactor Safety Study, an Assessment of Accident Risks. WASH 1400, Report NUREG-75/014. Washington, DC (USA): U.S. Nuclear Regulatory Commission; 1975.

6. USNRC. An approach for using probabilistic risk assessment in risk-informed decisions on plant-specific changes to the licensing basis. NUREG-1.174 - Revision 1. Washington, DC: US Nuclear Regulatory Commission; 2002.

7. USNRC. Guidance on the Treatment of Uncertainties Associated with PRAs in Risk-Informed Decision Making. NUREG-1855. Washington, DC: US Nuclear Regulatory Commission; 2009. 
8. U.S. NRC. Severe Accident Risks: An Assessment for Five U.S. Nuclear Power Plants. NUREG-1150, Vols. 1-3. Washington, DC: U.S. Nuclear Regulatory Commission, Office of Nuclear Regulatory Research, Division of Systems Research; 1990-1991.

9. Breeding RJ, Helton JC, Gorham ED, Harper FT. Summary Description of the Methods Used in the Probabilistic Risk Assessments for NUREG-1150. Nucl Eng Des 1992; 135(1): 1-27.

10. Helton JC, Breeding RJ. Calculation of Reactor Accident Safety Goals. Reliability Engineering and System Safety 1993; 39(2): 129-158.

11. Helton JC, Hansen CW, Swift PN, eds. Special Issue: Performance Assessment for the Proposed High-Level Radioactive Waste Repository at Yucca Mountain, Nevada. Reliability Engineering and System Safety 2014; 122: 1-456.

12. NAS/NRC (National Academy of Science/National Research Council). Evaluation of Quantification of Margins and Uncertainties for Assessing and Certifying the Reliability of the Nuclear Stockpile. Washington, DC: National Academy Press; 2008.

13. US EPA (Environmental Protection Agency). Guidance on the Development, Evaluation, and Application of Environmental Models. EPA/100/K-09/003. Washington, DC: U.S. Environmental Protection Agency, Council for Regulatory Environmental Modeling; 2009.

14. Helton JC, Hansen CW, Sallaberry CJ. Conceptual Structure and Computational Organization of the 2008 Performance Assessment for the Proposed High-Level Radioactive Waste Repository at Yucca Mountain, Nevada. Rel. Eng. Syst. Safety 2014; 122: 223-248.

15. Helton JC, Oberkampf W. Alternative representations of epistemic uncertainties. Reliability Engineering and System Safety 2004; 85(1-3): 1-10.

16. Helton JC, Johnson JD, Sallaberry CJ, Storlie CB. Survey of sampling-based methods for uncertainty and sensitivity analysis. Rel. Eng. Syst. Saf. 2006; 91: 1175-1209.

17. Helton JC, Davis FJ. Latin Hypercube Sampling and the Propagation of Uncertainty in Analyses of Complex Systems. Reliability Engineering and System Safety 2003; 81(1): 23-69.

18. Aven T. On the Need for Restricting the Probabilistic Analysis in Risk Assessments to Variability. Risk Analysis 2010; 30(3): 354-360.

19. Aven T. Interpretations of alternative uncertainty representations in a reliability and risk analysis context. Reliability Engineering \& System Safety 2011; 96(3): 353-360.

20. Aven T, Steen R. The concept of ignorance in a risk assessment and risk management context. Reliability Engineering and System Safety 2010; 95(11): 1117-1122. 
21. Aven T, Zio E. Some considerations on the treatment of uncertainties in risk assessment for practical decision making. Reliability Engineering and System Safety 2010; 96(1): 64-74.

22. Helton JC, Sallaberry CJ. Uncertainty and Sensitivity Analysis: From Regulatory Requirements to Conceptual Structure and Computational Implementation. IFIP Advances in Information and Communication Technology 2012; 377 AICT: 60-76.

23. Kennedy M, O’Hagan A. Bayesian calibration of computer models. Journal of the Royal Statistical Society, Series B 2001; 63: 425-464.

24. Bayarri MJ, Berger JO, Paulo R, Sacks J, Cafeo JA, Cavendish J, Lin CH, Tu J. A framework for validation of computer models. Technometrics 2007; 49: 138-154.

25. Oberkampf WL, Trucano TG. Verification and validation benchmarks. Nuclear Engineering and Design 2008; 238(3): 716-743.

26. Roy CJ, Oberkampf WL. A comprehensive framework for verification, validation, and uncertainty quantification in scientific computing. Computer Methods in Applied Mechanics and Engineering 2011; 200(25-28): 2131-2144.

27. Pasanisi A, Dutfoy A. An Industrial Viewpoint on Uncertainty Quantification in Simulation: Stakes, Methods, Tools, Examples. In: Dienstfrey AM, Boisvert RF, Ed. Uncertainty Quantification in Scientific Computing. Berlin (Germany): Springer; 2012. p. 27-45.

28. Aven T. Some reflections on uncertainty analysis and management. Reliability Engineering and System Safety 2010; 95: 195-201.

29. Dubois D, Guyonnet D. Risk-Informed Decision Making in the Presence of Epistemic Uncertainty. Int. J. General Systems 2011; 40(2): 145-167

30. Bernardo JM, Smith AFM. Bayesian Theory. Chichester (UK): Wiley; 1994.

31. Bedford T, Cooke R. Probabilistic Risk Analysis. Foundations and Methods. Cambridge (UK): Cambridge University Publishing Ltd; 2001.

32. Kelly DL, Smith CL. Bayesian Inference for Probabilistic Risk Assessment: A Practitioner's Guidebook. London (UK): Springer-Verlag; 2011.

33. Ferson S, Nelsen RB, Hajagos J, Berleant DJ, Zhang J, Tucker WT, Ginzburg LR, Oberkampf WL. Dependence in probabilistic modeling, Dempster-Shafer theory, and probability bounds analysis. Tech. Report SAND2004-3072. Albuquerque, NM: SANDIA National Labs; 2004.

34. Apostolakis GE, Kaplan S. Pitfalls in risk calculations. Reliability Engineering 1981; 2(2): 135-145.

35. Ferson S. What Monte Carlo methods cannot do. Hum Ecol Risk Assess 1996; 2: 990-1007. 
36. Ferson S, Burman MA. Correlation, dependency bounds and extinction risks. Biol. Conserv. 1995; 73: 101-105.

37. Ferson S, Ginzburg LR. Different methods are needed to propagate ignorance and variability. Reliability Engineering and Systems Safety 1996; 54: 133-144.

38. Helton JC, Johnson JD, Oberkampf WL. An exploration of alternative approaches to the representation of uncertainty in model predictions. Reliab Eng Syst Safe 2004; 85: 39-72.

39. Helton JC, Johnson JD. Quantification of Margins and Uncertainties: Alternative Representations of Epistemic Uncertainty. Reliab Eng Syst Safe 2011; 96(9): 1034-1052.

40. Klir GJ, Yuan B. Fuzzy Sets and Fuzzy Logic: Theory and Applications. Upper Saddle River, NJ: Prentice-Hall; 1995.

41. Beer M. Fuzzy probability theory. In: Meyers RA, Ed. Encyclopedia of Complexity and Systems Science - Vol.6. New York (NY): Springer; 2009. p. 4047-4059.

42. Molchanov I. Theory of Random Sets. New York (NY): Springer; 2005.

43. Ferson S, Kreinovich V, Ginzburg L, Sentz K, Myers DS. Constructing probability boxes and Dempster-Shafer structures. SAND2002-4015. Albuquerque, NM: SANDIA National Labs; 2003.

44. Helton J, Johnson JD, Oberkampf WL, Storlie CB. A sampling-based computational strategy for the representation of epistemic uncertainty in model predictions with evidence theory. Computer Methods in Applied Mechanics and Engineering 2007; 196: 3980-98.

45. Le Duy TD, Dieulle L, Vasseur D, Berenguer C, Couplet M. An alternative comprehensive framework using belief functions for parameter and model uncertainty analysis in nuclear probabilistic risk assessment applications. Proceedings of the Institution of Mechanical Engineers, Part O: Journal of Risk and Reliability 2013; 227(5): 471-490.

46. Sentz K, Ferson S. Combination of Evidence in Dempster-Shafer Theory. Technical Report SAND 2002-0835. Albuquerque, New Mexico: SANDIA National Laboratories; 2002.

47. Baudrit C, Dubois D. Practical Representations of Incomplete Probabilistic Knowledge. Computational Statistics \& Data Analysis 2006; 51(1): 86-108.

48. Baudrit C, Dubois D, Guyonnet D. Joint Propagation and Exploitation of Probabilistic and Possibilistic Information in Risk Assessment. IEEE Trans Fuzzy Syst 2006; 14(5): 593-608.

49. Baudrit C, Dubois D, Perrot N. Representing parametric probabilistic models tainted with imprecision. Fuzzy Sets and System 2008; 159(15): 1913-1928. 
50. Dubois D. Possibility Theory and Statistical Reasoning. Comput Stat Data An 2006; 51: $47-$ 69.

51. Crespo LG, Kenny SP, Giesy DP. Reliability analysis of polynomial systems subject to p-box uncertainties. Mechanical Systems and Signal Processing 2013; 37(1-2): 121-136.

52. Ferson S, Hajagos JG. Arithmetic with uncertain numbers: rigorous and (often) best possible answers. Reliability Engineering and System Safety 2004; 85: 135-152.

53. Ferson S, Kreinovich V, Hajagos J, Oberkampf W, Ginzburg L. Experimental Uncertainty Estimation and Statistics for Data Having Interval Uncertainty. Technical Report SAND20070939. Albuquerque, New Mewico: SANDIA National Laboratories; 2007.

54. Weichselberger K. The theory of interval-probability as a unifying concept for uncertainty. Int. J. Approx. Reasoning 2000; 24(2-3): 149-170.

55. Beer M, Ferson S. Special issue "Imprecise probabilities - What can they add to engineering analyses?”. Mechanical Systems and Signal Processing 2013; 37(1-2): 1-3.

56. Beer M, Ferson S, Kreinovich V. Imprecise probabilities in engineering analyses. Mechanical Systems and Signal Processing 2013; 37(1-2): 4-29.

57. Blockley D. Analysing uncertainties: Towards comparing Bayesian and interval probabilities. Mechanical Systems and Signal Processing 2013; 37(1-2): 30-42.

58. Pedroni N, Zio E. Empirical comparison of methods for the hierarchical propagation of hybrid uncertainty in risk assessment, in presence of dependences. International Journal of Uncertainty, Fuzziness and Knowledge-Based Systems 2012; 20(4): 509-557.

59. Pedroni N, Zio E. Uncertainty analysis in fault tree models with dependent basic events. Risk Analysis, an International Journal 2013; 33(6): 1146-73.

60. Pedroni N, Zio E, Ferrario E, Pasanisi A, Couplet M. Hierarchical propagation of probabilistic and non-probabilistic uncertainty in the parameters of a risk model. Computers and Structures 2013; 126: 199-213.

61. Pedroni N, Zio E, Pasanisi A, Couplet M. Empirical Comparison of Two Methods for the Bayesian Update of the Parameters of Probability Distributions in a Two-Level Hybrid Probabilistic-Possibilistic Uncertainty Framework for Risk Assessment. ASCE-ASME J. Risk Uncertainty Eng. Syst., Part A: Civ. Eng. 2016; 2(1): 04015015.

62. Limbourg P, de Rocquigny E. Uncertainty analysis using evidence theory - confronting level1 and level-2 approaches with data availability and computational constraints. Reliability Engineering and System Safety 2010; 95(5): 550-564. 
63. Parry GW. The Characterization of Uncertainty in Probabilistic Risk Assessments of Complex Systems. Reliability Engineering and System Safety 1996; 54(2-3): 119-126.

64. Hoffman FO, Hammonds JS. Propagation of Uncertainty in Risk Assessments: The Need to Distinguish Between Uncertainty Due to Lack of Knowledge and Uncertainty Due to Variability. Risk Analysis 1994; 14(5): 707-712.

65. Paté-Cornell ME. Uncertainties in Risk Analysis: Six Levels of Treatment. Reliability Engineering and System Safety 1996; 54(2-3): 95-111.

66. Helton JC, Davis FJ, Johnson JD. Characterization of Stochastic Uncertainty in the 1996 Performance Assessment for the Waste Isolation Pilot Plant. Reliability Engineering and System Safety 2000; 69(1-3): 167-189.

67. Helton JC, Gross MB, Hansen CW, Sallaberry CJ, Sevougian SD. Expected Dose for the Seismic Scenario Classes in the 2008 Performance Assessment for the Proposed High-Level Radioactive Waste Repository at Yucca Mountain, Nevada. Reliability Engineering and System Safety 2014; 122: 380-398.

68. US NRC. EPRI/NRC-RES Fire PRA methodology for nuclear power facilities, Volume 2: detailed methodology. Technical Report NUREG-CR-6850. Washington, DC: US Nuclear Regulatory Commission; 2005.

69. Helton JC, Martell M-A, Tierney MS. Characterization of Subjective Uncertainty in the 1996 Performance Assessment for the Waste Isolation Pilot Plant. Reliability Engineering and System Safety 2000; 69(1-3): 191-204.

70. Huang D, Chen T, Wang MT. A fuzzy set approach for event tree analysis. Fuzzy Sets and Systems 2001; 118: 153-165.

71. Krzykacz-Hausmann B. An approximate sensitivity analysis of results from complex computer models in the presence of epistemic and aleatory uncertainties. Reliab Eng Syst Safe 2006; 91: 1210-1218.

72. De Finetti B. Theory of Probability. New York, NY: Wiley; 1974.

73. Atwood CL, LaChance JL, Martz HF, Anderson DL, Englehardte M, Whitehead D, Wheeler T. Handbook of Parameter Estimation for Probabilistic Risk Assessment. Technical Report NUREG/CR-6823. Washington, DC: US Nuclear Regulatory Commission; 2003.

74. Frey HC, Burmaster DE. Methods for Characterizing Variability and Uncertainty: Comparison of Bootstrap Simulation and Likelihood-Based Approaches. Risk Anal 1999; 19(1): 109-130. 
75. Bergman B. Conceptualistic pragmatism: a framework for Bayesian analysis? IIE Transactions 2009; 41: 86-93.

76. Aven T, Heide B. Reliability and validity of risk analysis. Reliab Eng Syst Safe 2009; 94: $1862-1868$.

77. Dubois D. Representation, propagation and decision issues in risk analysis under incomplete probabilistic information. Risk Analysis 2010; 30: 361-368.

78. Helton JC, Pilch M, Sallaberry CJ. Probability of Loss of Assured Safety in Systems with Multiple Time-Dependent Failure Modes: Representations with Aleatory and Epistemic Uncertainty. Reliability Engineering and System Safety 2014; 124: 171-200.

79. Singpurwalla N. Reliability and Risk. A Bayesian Perspective. New York (NY): Wiley; 2006.

80. North W. Probability Theory and Consistent Reasoning. Risk Analysis 2010; 30(3): 377-380.

81. Cooke R. Experts in Uncertainty: Opinion and Subjective Probability in Science. Oxford; New York: Oxford University Press; 1991.

82. Ayyub BM. Elicitation of Expert Opinions for Uncertainty and Risks. Boca Raton, FL: CRC Press; 2001.

83. Hacking I. The Emergence of Probability: A Philosophical Study of Early Ideas About Probability, Induction and Statistical Inference. New York: Cambridge University Press; 1975.

84. Bernardo JM. The concept of exchangeability and its applications. Far East Journal of Mathematical Sciences 1996; 4: 111-121.

85. Shafer G. Perspectives on the theory and practice of belief functions. International Journal of Approximate Reasoning 1990; 4: 323-62.

86. Dempster AP. Upper and Lower Probability Inferences Based on a Sample from a Finite Univariate Population. Biometrika 1967; 54(2-3): 515-528.

87. Kozine I, Filimonov Y. Imprecise reliabilities: experiences and advances. Reliab. Eng. Syst. Saf. 2000; 67: 75-83.

88. Walley P. Statistical reasoning with imprecise probabilities. New York, NY: Chapman and Hall; 1991.

89. Lindley DV. Understanding uncertainty. Hoboken, NJ: Wiley; 2006.

90. Zadeh LA. Fuzzy sets as a basis for a theory of possibility. Fuzzy Set Syst 1978; 1: 3-28.

91. Karanki DR, Kushwaha HS, Verma AK, Ajit S. Uncertainty Analysis Based on Probability Bounds Approach in Probabilistic Safety Assessment. Risk Anal 2009; 29(5): 662-675. 
92. Zio E. The Monte Carlo Simulation Method for System Reliability and Risk Analysis. Springer Series in Reliability Engineering. London, UK: Springer; 2013.

93. Baraldi P, Zio E. A combined Monte Carlo and possibilistic approach to uncertainty propagation in event tree analysis. Risk Analysis 2008; 28(5): 1309-1325.

94. Baudrit C, Couso I, Dubois D. Joint propagation of probability and possibility in risk analysis: toward a formal framework. Internat. J. Approx. Reasoning 2007; 45(1): 82-105.

95. Baudrit C, Guyonnet D, Dubois D. Joint propagation of variability and imprecision in assessing the risk of groundwater contamination. J Contam Hydrol 2007; 93: 72-84.

96. Kentel E, Aral MM. Probabilistic-fuzzy health risk modeling. Stoch. Envir. Res. and Risk Ass. 2004; 18: pp. 324-338.

97. Zadeh LA. Fuzzy sets. Information and Control 1965; 8: 338-353.

98. Kentel E, Aral MM. 2D Monte Carlo versus 2D Fuzzy Monte Carlo Health Risk Assessment. Internat. J. Stochastic Environ. Res. Risk Assess. 2005; 19: 86-96.

99. Möller B, Beer M. Fuzzy Randomness: Uncertainty in Civil Engineering and Computational Mechanics. Berlin, Germany: Springer; 2004.

100. Moller B, Beer M, Graf W, Sickert JU. Time-dependent reliability of textile-strengthened RC structures under consideration of fuzzy randomness. Comput Struct 2006; 84: 585-603.

101. Fetz T, Oberguggenberger M. Propagation of uncertainty through multivariate functions in the framework of sets of probability measures. Reliab Eng Syst Safe 2004; 85: 73-87.

102. Tonon F. Using random set theory to propagate epistemic uncertainty through a mechanical system. Reliability Engineering and System Safety 2004; 85: 169-181.

103. Helton JC, Oberkampf WL, Johnson JD. Competing Failure Risk Analysis Using Evidence Theory. Risk Analysis 2005; 25(4): 973-995.

104. Siu N, Kelly D. Bayesian parameter estimation in probabilistic risk assessment. Reliability Engineering and System Safety 1998; 62: 89-116.

105. Lindley DV. The philosophy of statistics. The Statistician 2000; 49(3): 293-337.

106. Kelly DL, Smith CL. Bayesian inference in probabilistic risk assessment - The current state of the art. Reliability Engineering and System Safety 2009; 94: 628-643.

107. Pasanisi A, Keller M, Parent M. Estimation of a quantity of interest inuncertainty analysis: Some help from Bayesian decision theory. Reliab Eng Syst Safe 2012; 100: 93-101. 
108. Ferson S. Bayesian methods in risk assessment. Technical Report available at: www.ramas.com/bayes.pdf; 2005.

109. Smets P. Belief Functions: The Disjunctive Rule of Combination and the Generalized Bayesian Theorem. International Journal of Approximate Reasoning 1993; 9: 1-35.

110. Viertl R. Statistical Methods for Fuzzy Data. Chichester, UK: Wiley; 2011.

111. Viertl R, Hareter D. Generalized Bayes-theorem for non-precise a-priori distribution. Metrika 2004; 59(3): 263-273.

112. Dubois D, Prade H. Bayesian conditioning in possibility theory. Fuzzy Sets and Systems 1997; 92: 223-240.

113. Lapointe S, Bobeè B. Revision of possibility distributions: A Bayesian inference pattern. Fuzzy Sets and Systems 2000; 116: 119-140.

114. Stein M, Beer M, Kreinovich V. Bayesian Approach for Inconsistent Information. Information Sciences 2013; 245: 96-111.

115. US NRC. Common-cause failure database and analysis system: event data collection, Classification, and coding. NUREG/CR-6268. Washington, DC: US Nuclear Regulatory Commission; 2007.

116. Watts DJ. A Simple Model of Global Cascades on Random Networks. Proc. Natl. Acad. Sci. U.S.A. 2002; 99: 5766-5771.

117. Zio E, Sansavini G. Component Criticality in Failure Cascade Processes of Network Systems. Risk Analysis 2011; 31(8): 1196-1210.

118. Vaurio JK. Consistent mapping of common cause failure rates and alpha factors. Reliability Engineering and System Safety 2007; 92(5): 628-645.

119. Sadiq R, Saint-Martin E, Kleiner Y. Predicting risk of water quality failures in distribution networks under uncertainties using fault-tree analysis. Urban Water 2008; 5(4): 287-304.

120. Ferdous R, Khan F, Sadiq R, Amyotte P, Veitch B. Fault and Event Tree Analyses for Process Systems Risk Analysis: Uncertainty Handling Formulations. Risk Anal 2011; 31(1): 86-107.

121. Iman RL, Davenport JM. Rank Correlation Plots for Use with Correlated Input Variables. Communications in Statistics: Simulation and Computation 1982; B11(3): 335-360.

122. Zhang Q. A method dealing with correlations in uncertainty propagation by using traditional correlation coefficients. Reliability Engineering and System Safety 1993; 41(2): 107-114. 
123. Kafrawy KF, Rushdi AM. Uncertainty analysis of fault tree with statistically correlated failure data. Microelectronics and Reliability 1990; 30: 157-175.

124. Karanki DR, Jadhav PA, Chandrakar A, Srividya A, Verma AK. Uncertainty analysis in PSA with correlated input parameters. Int J Syst Assur Eng Manag 2010; 1: 66-71.

125. Williamson RC, Downs T. Probabilistic arithmetic I: Numerical methods for calculating convolutions and dependency bounds. Int J Approx Reason 1990; 4: 89-158.

126. Berleant D, Goodman-Strauss C. Bounding the results of arithmetic operations on random variables of unknown dependency using intervals. Reliable Computing 1998; 4: 147-165.

127. Berleant D, Zhang J. Representation and problem solving with Distribution Envelope Determination (DEnv). Reliability Engineering and System Safety 2004; 85: 153-168.

128. Berleant D, Xie L, Zhang J. Statool: a tool for distribution envelope determination, an intervalbased algorithm for arithmetic on random variables. Reliab Comput 2003; 9(2): 91-108.

129. Berleant D, Anderson G, Goodman-Strauss C. Arithmetic on Bounded Families of Distributions: A DEnv Algorithm Tutorial. In: Hu C et al., Eds. Knowledge Processing with Interval and Soft Computing. London, UK: Springer-Verlag; 2008. p. 183-210.

130. Dubois D, Prade H. When upper probabilities are possibility measures. Fuzzy Sets and Systems 1992; 49: 65-74.

131. Ralescu D. Average level of a fuzzy set. In: Bertoluzza C, Gil MA, Ralescu DA, eds. Statistical Modeling, Analysis and Management of Fuzzy Data. Berlin Heidelberg: Springer; 2002. p. 119-126.

132. Dubois D, Prade H, Sandri S. On possibility/probability transformations. In: Lowen R, Roubens M, eds. Fuzzy Logic: State of the Art. Dordrecht, the Netherlands: Kluwer Academic Publishers; 1993. p. 103-112.

133. Smets P. Constructing the pignistic probability function in a context of uncertainty. In: Henrion M, Shachter RD, Kanal LN, Lemmer JF, eds. Uncertainty in Artificial Intelligence 5. Amsterdam, The Netherlands: North-Holland; 1990. p. 29-39.

134. Dubois D, Foulloy L, Mauris G, Prade H. Probability-Possibility Transformation, Triangular Fuzzy Sets, and Probabilistic Inequalities. Reliable Computing 2004; 10: 273-297.

135. Flage R, Baraldi P, Zio E, Aven T. Probability and possibility-based representations of uncertainty in fault tree analysis. Risk Analysis 2013; 33(1): 121-33.

136. Aven T. On the use of conservatism in risk assessments. Reliability Engineering and System Safety 2016; 146: 33-38. 
137. Anoop MB, Balaji Rao K, Gopalakrishnan S. Conversion of probabilistic information into fuzzy sets for engineering decision analysis. Computers and Structures 2006; 84: 141-155.

138. Masson MH, Denoeux T. Inferring a possibility distribution from empirical data. Fuzzy Sets and Systems 2006; 157: 319-340.

139. Mauris G. Inferring a Possibility Distribution from Very Few Measurements. In: Dubois D et al., Eds. Soft Methods for Handling Variability and Imprecision, ASC 48. Berlin, Heidelberg: Springer-Verlag; 2008. p. 92-99.

\section{Acknowledgments}

The authors wish to thank the numerous different reviewers and the Area Editor, Prof. Seth Guikema, for their constructive comments that helped improving significantly the quality of the paper. 